\title{
Article \\ Reduced Chemical Kinetic Reaction Mechanism for Dimethyl Ether-Air Combustion
}

\author{
Niklas Zettervall ${ }^{1}$, Christer Fureby ${ }^{2}$ and Elna J. K. Nilsson ${ }^{3, *} \mathbb{C}$ \\ 1 Defense \& Security, Systems and Technology, Swedish Defense Research Agency, Tumba, \\ 14725 Stockholm, Sweden; niklas.zettervall@foi.se \\ 2 Department of Energy Sciences, Lund University, Box 118, 22100 Lund, Sweden; christer.fureby@energy.lth.se \\ 3 Combustion Physics, Lund University, Box 118, 22100 Lund, Sweden \\ * Correspondence: elna.heimdal_nilsson@forbrf.lth.se; Tel.: +46-222-14-03
}

check for updates

Citation: Zettervall, N.; Fureby, C.; Nilsson, E.J.K. Reduced Chemical Kinetic Reaction Mechanism for Dimethyl Ether-Air Combustion. Fuels 2021, 2, 323-344. https:// doi.org/10.3390/fuels2030019

Academic Editors: Olivier Mathieu and Nadir Yilmaz

Received: 2 March 2021

Accepted: 4 August 2021

Published: 25 August 2021

Publisher's Note: MDPI stays neutral with regard to jurisdictional claims in published maps and institutional affiliations.

Copyright: (c) 2021 by the authors. Licensee MDPI, Basel, Switzerland. This article is an open access article distributed under the terms and conditions of the Creative Commons Attribution (CC BY) license (https:// creativecommons.org/licenses/by/ $4.0 /)$.

\begin{abstract}
Development and validation of a new reduced dimethyl ether-air (DME) reaction mechanism is presented. The mechanism was developed using a modular approach that has previously been applied to several alkane and alkene fuels, and the present work pioneers the use of the modular methodology, with its underlying $\mathrm{H} / \mathrm{C}_{1} / \mathrm{O}$ base mechanism, on an oxygenated fuel. The development methodology uses a well-characterized $\mathrm{H} / \mathrm{C}_{1} / \mathrm{O}$ base mechanism coupled to a reduced set of fuel and intermediate product submechanisms. The mechanism for DME presented in this work includes 30 species and 69 irreversible reactions. When used in combustion simulation the mechanism accurately reproduced key combustion characteristics and the small size enables use in computationally demanding Large Eddy Simulations (LES) and Direct Numerical Simulations (DNS). It has been developed to accurately predict, among other parameters, laminar burning velocity and ignition delay times, including the negative temperature regime. The evaluation of the mechanism and comparison to experimental data and several detailed and reduced mechanisms covers a wide range of conditions with respect to temperature, pressure and fuel-to-air ratio. There is good agreement with experimental data and the detailed reference mechanisms at all investigated conditions. The mechanism uses fewer reactions than any previously presented DME-air mechanism, without losing in predictability.
\end{abstract}

Keywords: combustion chemistry; dimethyl ether; DME; reaction mechanism; reduced kinetics; biofuel combustion

\section{Introduction}

With the realization that global warming and air pollution as a result of fossil fuel combustion are increasing threats to human health and the environment, the demand for clean sources of energy is increasing. Biofuels represent an attractive alternative to the fossil hydrocarbon fuels that make up the vast majority of today's energy sources for transportation. Among biofuels with a potential to be extensively used in the near future, dimethyl ether, abbreviated as DME and with the molecular formula $\mathrm{CH}_{3} \mathrm{OCH}_{3}$, ranks highly according to some evaluations [1]. DME is the simplest ether compound, and from a molecular structure point of view, the absence of carbon-carbon bonds and the available oxygen in the molecular structure gives it advantageous characteristics for compression ignition combustion engines. From an environmental perspective, one of the important features is that this molecular structure does not promote $\mathrm{C}-\mathrm{C}$ recombination that could eventually result in soot formation.

DME has seen experimental use as an additive to the main fuel in diesel engines [2,3], with additions in the range of $10-30 \%$ DME by mass [4], or as a single fuel in diesel engines [5,6]. DME has also shown promise as a fuel in gas turbines [7-9] and as an addition to $n$-Butane in HCCI engines [10-12]. DME can either be produced from natural gas, coal or biomass [13] or via a dehydration reaction of methanol [14]. The high cetane 
number (>55) of DME allows long burning times, and compared to most other diesel engine fuels, this results in lower levels of $\mathrm{NO}_{x}, \mathrm{SO}_{x}$ and soot precursors $[2,3,6]$. DME has a relatively high reactivity resulting in ignition at low combustion temperatures [15]. The short auto-ignition times and the fact that it can be produced as a renewable fuel makes DME an attractive fuel, from both combustion technological and environmental standpoints.

One of the main advantages of using DME in diesel engines is its ignition characteristics where DME expresses a clear negative temperature coefficient (NTC) [16] behaviour represented by an increasing ignition time with increasing temperature within a specific low- and medium-temperature range. The temperature at which the NTC behaviour starts is dependent on both the pressure and mixture composition [17], where increasing pressure or an increasing equivalence ratio will shift the NTC region towards higher temperatures. Increasing pressure will also lower the ignition delay time, mainly due to the increase in reactants and radicals in the mixture [17].

Combustion research often includes experimental as well as computational studies to get a comprehensive understanding of the combustion processes [18]. This includes understanding processes on a wide range of both length and time scales, from the chemical reactivity on molecular levels all the way to the scales present in the physical phenomena governing the turbulent flow. Laminar premixed flames represent a fundamental flame characteristic of a premixed fuel-air mixture. Laminar burning velocity, $\mathrm{S}_{\mathrm{L}}$, is a key parameter for premixed flows and can help predict flashback, dynamic instabilities and blow-off events in gas turbine engines $[19,20]$. This means that the laminar burning velocity represents a fundamental development and validation target when modelling the combustion process using a kinetic mechanism. Several experimental studies on laminar burning velocity for DME have been made over the years [21-26], at both ambient (1 atm) and elevated pressures (5,10 atm).

Detailed chemical kinetic reaction mechanisms on DME-air combustion have been developed by Zhao et al. [27], Fischer et al. [28,29] and Burke et al. [17]. The two latter mechanisms and their respective validation targets are outlined in Table 1, and both include a high chemical complexity with both low- and high-temperature kinetics capable of modelling flames for a wide range of conditions. Other detailed DME-air reaction mechanisms exist, but in order to limit the comparison, three detailed reaction mechanisms are included $[17,28,30]$. These three detailed mechanisms provide a good platform when representing different versions of the DME chemistry, with the mechanism by Burke et al. [17] offering by far the most detailed description. These three mechanisms include a wide range of number of species (48 to 433 ) and reactions (284 to 1004) yet can all be classified as chemically complex. They cover the modelling of the laminar burning velocity, major species concentrations and ignition delay time, for a wide range of equivalence ratios, pressures and temperatures. These combustion parameters, at these wide ranges of conditions, are all targets that the novel reduced mechanism presented here aim to model, making these three detailed mechanisms suitable as references to compare to. For a more detailed list of which combustion parameter each of these three reference mechanisms cover see Table 1. Several reduced DME-air mechanisms exist [31-33], varying in size from 26 to 41 species and 66 (reversible) to 110 (reversible) reactions. Again, to limit the comparison, two [32,33] out of these three reduced mechanisms were chosen in the comparison below, with the mechanism by Khare et al. [32] being the smallest of the three (using both reversible and irreversible reactions) and the one by Pan et al. [33] (using reversible reactions) representing a mechanism of intermediate size. The mechanism by Khare et al. [32] is based on the San Diego mechanism [30]. They have been validated for both laminar flames [32] and shock tube experiments [32,33]. 
Table 1. Reaction mechanisms. Abbreviations of validation targets: first part indicates experimental configuration (JSR - Jet Stirred Reactor, ST—Shock Tube, FR-Flow Reactor, LF-Laminar Flame); second part is type of data (ig-ignition delay time, $\mathrm{S}_{\mathrm{L}}$-laminar burning velocity, s-speciation, ext-extinction strain rate). The San Diego, LLNL and Zhao290 reaction mechanisms use only reversible reactions whereas Aramco, P82 and K71 use both reversible and irreversible reactions.

\begin{tabular}{ccccc}
\hline & Reference & Species & Reactions & Validation Targets \\
\hline Aramco & {$[17]$} & 433 & 1004 & FR, JSR, ST-ig, LF-S, LF-s \\
LLNL & {$[28]$} & 79 & 697 & JSR, ST, LF \\
San Diego & {$[30]$} & 48 & 284 & JSR, ST, LF \\
P82 & {$[33]$} & 27 & 82 & ST-ig, engine emission data \\
K71 & {$[32]$} & 26 & 71 & ST-ig, LF-ext \\
\hline
\end{tabular}

Ever increasing development in computational capacities has enabled the use of Large Eddy Simulations (LES) to become a viable simulation option for a wide range of applications [34-36]. That said the computational capacity still represents a major limiting factor with geometric representation, applied simulation models, number of mesh cells and the complexity and numerical stiffness of the chemistry determining the computational cost. One of the main limiting factors to the overall computational cost is the cost related to the modelling of the chemical kinetics hence several reduction techniques have been developed over the years [37-39]. The high computational cost effectively excludes use of any of the detailed reaction mechanisms due to their high computational cost, creating a demand for smaller reaction mechanisms. Several high-fidelity combustion LES studies, incorporating reduced kinetic mechanisms of hydrogen and a range of hydrocarbons, have been published by Bulat et al. [40], Zettervall et al. [36,41-43], Liu et al. [44], Fureby [45], Larsson et al. [35], Vincent-Randonnier et al. [46,47] and Danel et al. [48].

In the present work we present a new reduced reaction mechanism for DME combustion, specifically developed for use in combustion LES. Several parameters need to be met before the mechanism can be successfully implemented in high-fidelity LES, such as having a low computational cost, determined by mainly number of species and reactions, and the numerical stiffness of the mechanism itself. A low number of species will also require less memory storage in a LES. The mechanism is developed to accurately predict combustion characteristics over a wide range of temperature, pressure and fuel/oxidizer ratio conditions. The presented reaction mechanism is then compared to experimental data and several available detailed and reduced reaction mechanisms for DME combustion.

\section{Mechanism Development}

\subsection{Mechanism Development Methodology}

The reduced reaction mechanism presented here builds on the well-proven mechanism build-up concept used by Zettervall et al. [36] on hydrogen $\left(\mathrm{H}_{2}\right)$, Larsson et al. [35] on methane $\left(\mathrm{CH}_{4}\right)$, and Zettervall et al. [49] on ethylene $\left(\mathrm{C}_{2} \mathrm{H}_{4}\right)$, propane $\left(\mathrm{C}_{3} \mathrm{H}_{8}\right)$ [43] and kerosene $\left(\mathrm{C}_{12} \mathrm{H}_{23}\right)[41,50,51]$.

A thorough description of the development process is given by Zettervall et al. [50]. The reduced mechanism can be sectioned into three subsets: fuel breakdown, intermediate hydrocarbon oxidation and base mechanism. The previous ethylene [49], propane [43] and kerosene mechanisms $[41,50,51]$ and the DME reaction mechanism presented here all use the same set of reactions in their respective $\mathrm{H} / \mathrm{C}_{1} / \mathrm{O}$ base mechanism. The intermediate hydrocarbon oxidation submechanism presented here however differs to previous work on the $C_{2}-C_{12}$ species fuels [43,49-51]. The intermediate hydrocarbon oxidation section is radically different in order to facilitate the important low- and medium-temperature kinetics that accompany oxygenated biofuels in general and DME in particular. It was found, during the development work, that adding reactions for $\mathrm{C}_{2} \mathrm{H}_{\mathrm{x}}$ species did not affect the overall predictability of the flame parameters targeted, hence these species and reactions were not included in order to keep the number of species and reactions in the mechanism to a minimum. The basic structure of the mechanism is shown in Figure 1. 
Mechanism structure, Z69.

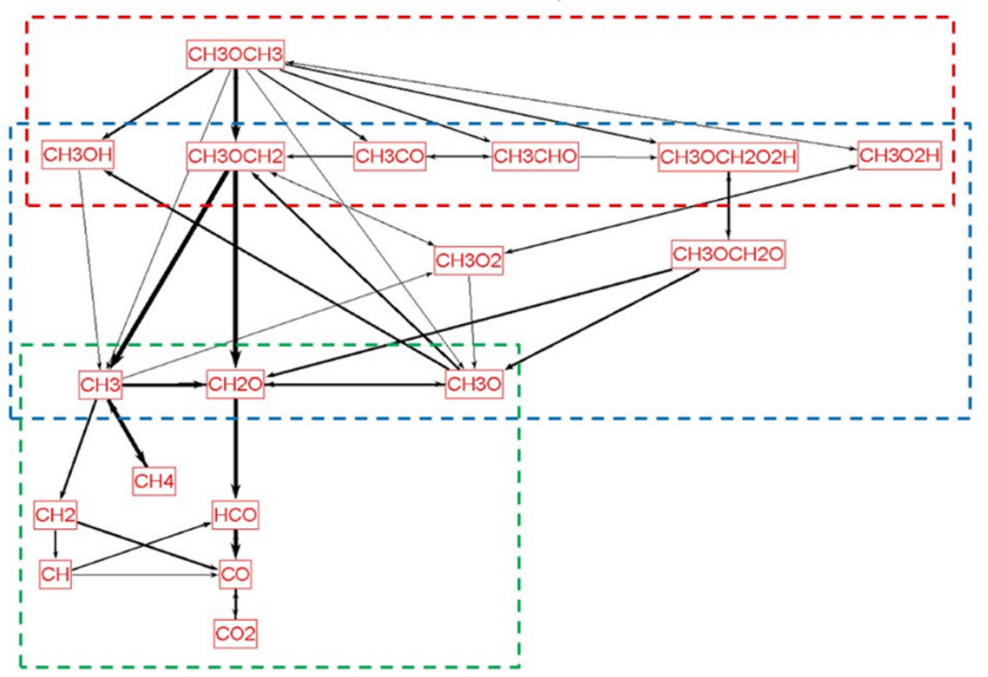

Figure 1. Mechanism structure diagram for Z69. The coloured boxes refer to the three submechanisms-base mechanism in green, intermediate hydrocarbon oxidation in blue and fuel breakdown reactions in red.

For fuel relevant reactions for this DME mechanism mainly the two detailed mechanisms by Fischer et al. [28] and Burke et al. [17] were used as the starting point. Combustion characteristics obtained from experiments and detailed reaction mechanisms then guided the development process, which included minor adjustments to reaction rate parameters for reactions in the reduced reaction mechanism. Key flame properties guiding the development are the laminar burning velocity, $\mathrm{S}_{\mathrm{L}}$, at different initial gas temperatures and pressures, flame temperature, $\mathrm{T}_{\text {flame }}$, profiles of major species $\left(\mathrm{CO}, \mathrm{CO}_{2}, \mathrm{H}_{2} \mathrm{O}, \mathrm{H}_{2}\right)$, key minor species $\left(\mathrm{CH}_{3} \mathrm{O}, \mathrm{CH}_{2} \mathrm{O}, \mathrm{OH}, \mathrm{O}\right)$ as well as the ignition delay times, $\tau_{\mathrm{ig}}$, and extinction strain rates, $\sigma_{\text {ext }}$, all for the relevant range of temperatures, pressures and equivalence ratios. These flame properties thoroughly characterize a flame; predicting them at a wide range of conditions ensures that the reaction mechanism is versatile and that it can be applied for a wide range of flame configurations.

The experimental data and reference simulations used as reference targets are summarized in Table 2. The reduced reaction mechanism presented in this work consists of 30 species and 69 irreversible reactions, and the mechanism is listed in Table 3 and will from now on be referred to as Z69.

Table 2. Summary of experimental data and modelling performed using the reference mechanisms in their respective studies.

\begin{tabular}{cccccccc}
\hline & \multicolumn{3}{c}{ Experiments } & \multicolumn{3}{c}{ Reference Simulations } \\
\hline Type & $\mathbf{P}(\mathbf{a t m})$ & $\mathbf{T}(\mathbf{K})$ & $\boldsymbol{\phi}$ & Ref. & $\mathbf{P}(\mathbf{a t m})$ & $\mathbf{T}(\mathbf{K})$ & $\boldsymbol{\phi}$ \\
\hline Laminar flames & $1-10$ & 298 & $0.7-1.7$ & {$[23]$} & $1-10$ & $300-750$ & $0.5-1.8$ \\
& $1-10$ & 298 & $0.7-1.6$ & {$[26]$} & & & \\
& 1 & 293 & $0.6-1.6$ & {$[25]$} & & & \\
& 1 & 298 & $0.7-1.8$ & {$[21]$} & & & \\
Ignition delay & 1 & 298 & $0.7-1.4$ & {$[24]$} & & & \\
& 1 & 298 & $0.7-1.4$ & {$[22]$} & & & \\
& $2-10$ & $700-1400$ & $0.5-1.0$ & {$[15]$} & $2-25$ & $600-1500$ & $0.5-2.0$ \\
Extinction strain rate & $10-25$ & $630-1450$ & $0.5-2.0$ & {$[17]$} & & & \\
& & & & & $1-10$ & 300 & 1.0 \\
\hline
\end{tabular}


Table 3. The reduced reaction mechanism $\mathrm{Z} 69\left(\mathrm{k}=\mathrm{A} \times \mathrm{T}^{\mathrm{n}} \times \exp \left(-\mathrm{E}_{\mathrm{a}} / \mathrm{RT}\right)\right.$, units: $\left.\mathrm{s}, \mathrm{mole}, \mathrm{cm}^{3}, \mathrm{cal}, \mathrm{K}\right)$.

\begin{tabular}{|c|c|c|c|c|c|}
\hline & Reaction & A & $\mathbf{N}$ & $\mathbf{E}_{\mathbf{a}}$ & Ref. \\
\hline \multirow[t]{3}{*}{1} & $\mathrm{CH}_{3} \mathrm{OCH}_{3}+\mathrm{M} \rightarrow \mathrm{CH}_{3}+\mathrm{CH}_{3} \mathrm{O}+\mathrm{M}^{\mathrm{f}}$ & & & & [17] \\
\hline & $\mathrm{k}_{\mathrm{f}}$ & $4.38 \times 10^{21}$ & -1.57 & 83,890 & \\
\hline & $\mathrm{k}_{\mathrm{f} 0}$ & $7.52 \times 10^{15}$ & 0 & 42,790 & \\
\hline 2 & $\mathrm{CH}_{3} \mathrm{OCH}_{3}+\mathrm{OH} \rightarrow \mathrm{CH}_{3} \mathrm{OCH}_{2}+\mathrm{H}_{2} \mathrm{O}$ & $6.32 \times 10^{6}$ & 2.00 & -652 & [17] \\
\hline 3 & $\mathrm{CH}_{3} \mathrm{OCH}_{3}+\mathrm{H} \rightarrow \mathrm{CH}_{3} \mathrm{OCH}_{2}+\mathrm{H}_{2}$ & $7.72 \times 10^{6}$ & 2.09 & 3384 & [17] \\
\hline 4 & $\mathrm{CH}_{3} \mathrm{OCH}_{3}+\mathrm{O} \rightarrow \mathrm{CH}_{3} \mathrm{OCH}_{2}+\mathrm{OH}$ & $7.75 \times 10^{8}$ & 1.36 & 2250 & [17] \\
\hline 5 & $\mathrm{CH}_{3} \mathrm{OCH}_{3}+\mathrm{CH}_{3} \mathrm{O}_{2} \rightarrow \mathrm{CH}_{3} \mathrm{OCH}_{2}+\mathrm{CH}_{3} \mathrm{O}_{2} \mathrm{H}$ & $1.68 \times 10^{13}$ & 0 & 13,690 & [28] \\
\hline 6 & $\mathrm{CH}_{3} \mathrm{OCH}_{3}+\mathrm{CH}_{3} \mathrm{CO} \rightarrow \mathrm{CH}_{3} \mathrm{OCH}_{2}+\mathrm{CH}_{3} \mathrm{CHO}$ & $7.75 \times 10^{11}$ & 0.28 & 16,980 & {$[28]$} \\
\hline 7 & $\mathrm{CH}_{3} \mathrm{OCH}_{3}+\mathrm{CH}_{3} \mathrm{O} \rightarrow \mathrm{CH}_{3} \mathrm{OCH}_{2}+\mathrm{CH}_{3} \mathrm{OH}$ & $6.02 \times 10^{11}$ & 0 & 4074 & [28] \\
\hline 8 & $\mathrm{CH}_{3} \mathrm{OCH}_{3}+\mathrm{O}_{2} \rightarrow \mathrm{CH}_{3} \mathrm{OCH}_{2}+\mathrm{HO}_{2}$ & $4.10 \times 10^{13}$ & 0 & 44,910 & [17] \\
\hline 9 & $\mathrm{CH}_{3} \mathrm{OCH}_{2}+\mathrm{CH}_{3} \mathrm{O}_{2} \mathrm{H} \rightarrow \mathrm{CH}_{3} \mathrm{OCH}_{3}+\mathrm{CH}_{3} \mathrm{O}_{2}$ & $2.29 \times 10^{14}$ & -0.8 & 7270 & [28] \\
\hline 10 & $\mathrm{CH}_{3} \mathrm{OCH}_{2} \rightarrow \mathrm{CH}_{2} \mathrm{O}+\mathrm{CH}_{3}$ & $1.60 \times 10^{13}$ & 0 & 25,500 & [17] \\
\hline 11 & $\mathrm{CH}_{3} \mathrm{OCH}_{2}+\mathrm{O}_{2} \rightarrow \mathrm{CH}_{3} \mathrm{OCH}_{2} \mathrm{O}_{2}$ & $2.00 \times 10^{12}$ & 0 & 0 & [17] \\
\hline 12 & $\mathrm{CH}_{3} \mathrm{OCH}_{2} \mathrm{O}_{2}+\mathrm{CH}_{3} \mathrm{OCH}_{2} \mathrm{O}_{2} \rightarrow \mathrm{O}_{2}+\mathrm{CH}_{3} \mathrm{OCH}_{2} \mathrm{O}+\mathrm{CH}_{3} \mathrm{OCH}_{2} \mathrm{O}$ & $1.55 \times 10^{23}$ & -4.5 & 0 & [17] \\
\hline 13 & $\mathrm{CH}_{3} \mathrm{OCH}_{2} \mathrm{O}_{2} \rightarrow \mathrm{CH}_{3} \mathrm{OCH}_{2}+\mathrm{O}_{2}$ & $4.44 \times 10^{19}$ & -1.59 & 36,240 & [28] \\
\hline 14 & $\mathrm{CH}_{3} \mathrm{OCH}_{2} \mathrm{O}_{2}+\mathrm{CH}_{3} \mathrm{CHO} \rightarrow \mathrm{CH}_{3} \mathrm{OCH}_{2} \mathrm{O}_{2} \mathrm{H}+\mathrm{CH}_{3} \mathrm{CO}$ & $2.80 \times 10^{12}$ & 0 & 13,600 & [17] \\
\hline 15 & $\mathrm{CH}_{3} \mathrm{OCH}_{2} \mathrm{O}_{2}+\mathrm{CH}_{3} \mathrm{OCH}_{3} \rightarrow \mathrm{CH}_{3} \mathrm{OCH}_{2} \mathrm{O}_{2} \mathrm{H}+\mathrm{CH}_{3} \mathrm{CO}+\mathrm{H}_{2}$ & $2.80 \times 10^{12}$ & 0 & 10,600 & a \\
\hline 16 & $\mathrm{CH}_{3} \mathrm{OCH}_{2} \mathrm{O}_{2} \rightarrow \mathrm{CH}_{2} \mathrm{OCH}_{2} \mathrm{O}_{2} \mathrm{H}$ & $2.20 \times 10^{9}$ & 0 & 10,846 & [32] \\
\hline 17 & $\mathrm{CH}_{2} \mathrm{OCH}_{2} \mathrm{O}_{2} \mathrm{H} \rightarrow \mathrm{CH}_{3} \mathrm{OCH}_{2} \mathrm{O}_{2}$ & $2.20 \times 10^{9}$ & 0 & 500 & a \\
\hline 18 & $\mathrm{CH}_{2} \mathrm{OCH}_{2} \mathrm{O}_{2} \mathrm{H} \rightarrow 2 \mathrm{CH}_{2} \mathrm{O}+\mathrm{OH}$ & $1.50 \times 10^{13}$ & 0 & 30,500 & [32], c \\
\hline 19 & $\mathrm{CH}_{2} \mathrm{OCH}_{2} \mathrm{O}_{2} \mathrm{H}+\mathrm{O}_{2} \rightarrow \mathrm{HO}_{2} \mathrm{CH}_{2} \mathrm{OCHO}+\mathrm{OH}$ & $2.86 \times 10^{16}$ & -1.48 & 1873 & [32] \\
\hline 20 & $\mathrm{HO}_{2} \mathrm{CH}_{2} \mathrm{OCHO} \rightarrow \mathrm{CH}_{2} \mathrm{O}+\mathrm{CO}_{2}+\mathrm{OH}+\mathrm{H}$ & $2.50 \times 10^{16}$ & 0 & 43,000 & [32] \\
\hline 21 & $\mathrm{HO}_{2} \mathrm{CH}_{2} \mathrm{OCHO} \rightarrow \mathrm{CH}_{2} \mathrm{O}+\mathrm{CO}+2 \mathrm{OH}$ & $2.50 \times 10^{16}$ & 0 & 38,000 & [32], c \\
\hline 22 & $\mathrm{CH}_{3} \mathrm{OCH}_{2} \mathrm{O}+\mathrm{OH} \rightarrow \mathrm{CH}_{3} \mathrm{OCH}_{2} \mathrm{O}_{2} \mathrm{H}$ & $2.00 \times 10^{13}$ & 0 & 0 & [28] \\
\hline 23 & $\mathrm{CH}_{3} \mathrm{OCH}_{2} \mathrm{O} \rightarrow \mathrm{CH}_{3} \mathrm{O}+\mathrm{CH}_{2} \mathrm{O}$ & $5.18 \times 10^{12}$ & -0.13 & 19,370 & [28] \\
\hline 24 & $\mathrm{CH}_{3} \mathrm{OCH}_{2} \mathrm{O}_{2} \mathrm{H} \rightarrow \mathrm{CH}_{3} \mathrm{OCH}_{2} \mathrm{O}+\mathrm{OH}$ & $4.38 \times 10^{21}$ & -1.94 & 41,700 & {$[28], \mathrm{c}$} \\
\hline 25 & $\mathrm{CH}_{3} \mathrm{O}_{2}+\mathrm{CH}_{3} \mathrm{O}_{2} \rightarrow \mathrm{O}_{2}+\mathrm{CH}_{3} \mathrm{O}+\mathrm{CH}_{3} \mathrm{O}$ & $1.40 \times 10^{16}$ & -1.61 & 1860 & {$[28]$} \\
\hline \multirow[t]{3}{*}{26} & $\mathrm{CH}_{3} \mathrm{OH}+\mathrm{M} \rightarrow \mathrm{CH}_{3}+\mathrm{OH}+\mathrm{Mg}$ & & & & [28] \\
\hline & $\mathrm{k}_{\mathrm{f}}$ & $1.90 \times 10^{16}$ & 0 & 91,730 & \\
\hline & $\mathrm{k}_{\mathrm{f} 0}$ & $2.95 \times 10^{44}$ & -7.35 & 95,460 & \\
\hline 27 & $\mathrm{CH}_{3}+\mathrm{O}_{2}+\mathrm{M} \rightarrow \mathrm{CH}_{3} \mathrm{O}_{2}+\mathrm{M}$ & $2.40 \times 10^{13}$ & 0 & 28,812 & a \\
\hline \multirow[t]{3}{*}{28} & $\mathrm{CH}_{4}(+\mathrm{M}) \rightarrow \mathrm{CH}_{3}+\mathrm{H}(+\mathrm{M})^{\mathrm{d}}$ & & & & [35] \\
\hline & $\mathrm{k}_{\mathrm{f}}$ & $6.30 \times 10^{14}$ & 0 & 104,000 & \\
\hline & $\mathrm{k}_{\mathrm{f} 0}$ & $1.00 \times 10^{17}$ & 0 & 86,000 & \\
\hline \multirow[t]{3}{*}{29} & $\mathrm{CH}_{3}+\mathrm{H}(+\mathrm{M}) \rightarrow \mathrm{CH}_{4}(+\mathrm{M})^{\mathrm{d}}$ & & & & [35] \\
\hline & $\mathrm{k}_{\mathrm{f}}$ & $5.20 \times 10^{12}$ & 0 & -1310 & \\
\hline & $\mathrm{k}_{\mathrm{f} 0}$ & $8.25 \times 10^{14}$ & 0 & $-19,310$ & \\
\hline 30 & $\mathrm{CH}_{4}+\mathrm{H} \rightarrow \mathrm{CH}_{3}+\mathrm{H}_{2}$ & $2.20 \times 10^{4}$ & 3 & 8750 & [35] \\
\hline 31 & $\mathrm{CH}_{3}+\mathrm{H}_{2} \rightarrow \mathrm{CH}_{4}+\mathrm{H}$ & $9.57 \times 10^{2}$ & 3 & 8750 & [35] \\
\hline 32 & $\mathrm{CH}_{4}+\mathrm{OH} \rightarrow \mathrm{CH}_{3}+\mathrm{H}_{2} \mathrm{O}$ & $1.60 \times 10^{6}$ & 2.1 & 2460 & {$[35]$} \\
\hline 33 & $\mathrm{CH}_{3}+\mathrm{H}_{2} \mathrm{O} \rightarrow \mathrm{CH}_{4}+\mathrm{OH}$ & $3.02 \times 10^{5}$ & 2.1 & 17,422 & [35] \\
\hline 34 & $\mathrm{CH}_{3}+\mathrm{O} \rightarrow \mathrm{CH}_{2} \mathrm{O}+\mathrm{H}$ & $6.80 \times 10^{13}$ & 0 & 0 & [35] \\
\hline 35 & $\mathrm{CH}_{3}+\mathrm{O}_{2} \rightarrow \mathrm{CH}_{3} \mathrm{O}+\mathrm{O}$ & $3.00 \times 10^{13}$ & 0 & 25,652 & [35], b \\
\hline 36 & $\mathrm{CH}_{3}+\mathrm{OH} \rightarrow \mathrm{CH}_{2}+\mathrm{H}_{2} \mathrm{O}$ & $7.60 \times 10^{6}$ & 2 & 5000 & [35] \\
\hline 37 & $\mathrm{CH}_{3} \mathrm{O}+\mathrm{H} \rightarrow \mathrm{CH}_{2} \mathrm{O}+\mathrm{H}_{2}$ & $2.00 \times 10^{13}$ & 0 & 0 & [35] \\
\hline 38 & $\mathrm{CH}_{3} \mathrm{O}+\mathrm{M} \rightarrow \mathrm{CH}_{2} \mathrm{O}+\mathrm{H}+\mathrm{M}$ & $2.40 \times 10^{13}$ & 0 & 28,812 & [35] \\
\hline 39 & $\mathrm{CH}_{2}+\mathrm{O} \rightarrow \mathrm{CO}+\mathrm{H}_{2}$ & $3.00 \times 10^{13}$ & 0 & 0 & [35] \\
\hline 40 & $\mathrm{CH}_{2}+\mathrm{OH} \rightarrow \mathrm{CH}+\mathrm{H}_{2} \mathrm{O}$ & $1.13 \times 10^{7}$ & 2 & 3000 & {$[35], \mathrm{b}$} \\
\hline 41 & $\mathrm{CH}_{2} \mathrm{O}+\mathrm{H} \rightarrow \mathrm{HCO}+\mathrm{H}_{2}$ & $5.00 \times 10^{13}$ & 0 & 3991 & [35], b \\
\hline 42 & $\mathrm{CH}_{2} \mathrm{O}+\mathrm{OH} \rightarrow \mathrm{HCO}+\mathrm{H}_{2} \mathrm{O}$ & $1.40 \times 10^{14}$ & 0 & 1100 & [35], b, c \\
\hline 43 & $\mathrm{CH}+\mathrm{O} \rightarrow \mathrm{CO}+\mathrm{H}$ & $5.70 \times 10^{13}$ & 0 & 0 & [35] \\
\hline 44 & $\mathrm{CH}+\mathrm{OH} \rightarrow \mathrm{HCO}+\mathrm{H}$ & $3.00 \times 10^{13}$ & 0 & 0 & [35] \\
\hline 45 & $\mathrm{CH}+\mathrm{O}_{2} \rightarrow \mathrm{HCO}+\mathrm{O}$ & $3.30 \times 10^{13}$ & 0 & 0 & [35] \\
\hline 46 & $\mathrm{CH}+\mathrm{CO}_{2} \rightarrow \mathrm{HCO}+\mathrm{CO}$ & $8.40 \times 10^{13}$ & 0 & 200 & [35] \\
\hline 47 & $\mathrm{HCO}+\mathrm{H} \rightarrow \mathrm{CO}+\mathrm{H}_{2}$ & $4.00 \times 10^{13}$ & 0 & 0 & [35] \\
\hline 48 & $\mathrm{HCO}+\mathrm{M} \rightarrow \mathrm{CO}+\mathrm{H}+\mathrm{M}$ & $1.60 \times 10^{14}$ & 0 & 14,700 & {$[35]$} \\
\hline
\end{tabular}


Table 3. Cont.

\begin{tabular}{|c|c|c|c|c|c|}
\hline & Reaction & A & $\mathbf{N}$ & $\mathbf{E}_{\mathbf{a}}$ & Ref. \\
\hline 49 & $\mathrm{CO}+\mathrm{OH} \rightarrow \mathrm{CO}_{2}+\mathrm{H}$ & $1.51 \times 10^{7}$ & 1.3 & -758 & [35] \\
\hline 50 & $\mathrm{CO}_{2}+\mathrm{H} \rightarrow \mathrm{CO}+\mathrm{OH}$ & $1.57 \times 10^{9}$ & 1.3 & 20,400 & [35], c \\
\hline 51 & $\mathrm{H}+\mathrm{O}_{2} \rightarrow \mathrm{OH}+\mathrm{O}$ & $1.50 \times 10^{14}$ & 0 & 16,800 & {$[35], b$} \\
\hline 52 & $\mathrm{OH}+\mathrm{O} \rightarrow \mathrm{H}+\mathrm{O}_{2}$ & $1.20 \times 10^{13}$ & 0 & 690 & [35] \\
\hline 53 & $\mathrm{O}+\mathrm{H}_{2} \rightarrow \mathrm{OH}+\mathrm{H}$ & $1.80 \times 10^{10}$ & 1 & 8826 & [35] \\
\hline 54 & $\mathrm{OH}+\mathrm{H} \rightarrow \mathrm{O}+\mathrm{H}_{2}$ & $8.00 \times 10^{9}$ & 1 & 6760 & [35] \\
\hline 55 & $\mathrm{H}_{2}+\mathrm{OH} \rightarrow \mathrm{H}_{2} \mathrm{O}+\mathrm{H}$ & $1.17 \times 10^{9}$ & 1.3 & 3626 & [35] \\
\hline 56 & $\mathrm{H}_{2} \mathrm{O}+\mathrm{H} \rightarrow \mathrm{H}_{2}+\mathrm{OH}$ & $6.00 \times 10^{9}$ & 1.3 & 18,588 & {$[35], b$} \\
\hline 57 & $\mathrm{OH}+\mathrm{OH} \rightarrow \mathrm{O}+\mathrm{H}_{2} \mathrm{O}$ & $6.00 \times 10^{8}$ & 1.3 & 0 & [35] \\
\hline 58 & $\mathrm{O}+\mathrm{H}_{2} \mathrm{O} \rightarrow \mathrm{OH}+\mathrm{OH}$ & $4.00 \times 10^{9}$ & 1.3 & 17,029 & {$[35], b$} \\
\hline 59 & $\mathrm{H}+\mathrm{O}_{2}+\mathrm{M} \rightarrow \mathrm{HO}_{2}+\mathrm{M}^{\mathrm{e}}$ & $2.20 \times 10^{18}$ & -0.8 & 0 & {$[35], b$} \\
\hline 60 & $\mathrm{H}+\mathrm{HO}_{2} \rightarrow \mathrm{OH}+\mathrm{OH}$ & $1.50 \times 10^{14}$ & 0 & 1004 & [35] \\
\hline 61 & $\mathrm{H}+\mathrm{HO}_{2} \rightarrow \mathrm{H}_{2}+\mathrm{O}_{2}$ & $2.50 \times 10^{13}$ & 0 & 700 & [35] \\
\hline 62 & $\mathrm{OH}+\mathrm{HO}_{2} \rightarrow \mathrm{H}_{2} \mathrm{O}+\mathrm{O}_{2}$ & $2.00 \times 10^{13}$ & 0 & 1000 & [35] \\
\hline 63 & $\mathrm{HO}_{2}+\mathrm{HO}_{2} \rightarrow \mathrm{H}_{2} \mathrm{O}_{2}+\mathrm{O}_{2}$ & $2.00 \times 10^{14}$ & 0 & 0 & {$[35], b$} \\
\hline 64 & $\mathrm{H}_{2} \mathrm{O}_{2}+\mathrm{M} \rightarrow \mathrm{OH}+\mathrm{OH}+\mathrm{M}$ & $1.30 \times 10^{17}$ & 0 & 45,500 & [35] \\
\hline 65 & $\mathrm{OH}+\mathrm{OH}+\mathrm{M} \rightarrow \mathrm{H}_{2} \mathrm{O}_{2}+\mathrm{M}$ & $9.86 \times 10^{14}$ & 0 & -5070 & [35] \\
\hline 66 & $\mathrm{H}_{2} \mathrm{O}_{2}+\mathrm{OH} \rightarrow \mathrm{H}_{2} \mathrm{O}+\mathrm{HO}_{2}$ & $1.00 \times 10^{13}$ & 0 & 1800 & [35] \\
\hline 67 & $\mathrm{H}_{2} \mathrm{O}+\mathrm{HO}_{2} \rightarrow \mathrm{H}_{2} \mathrm{O}_{2}+\mathrm{OH}$ & $2.86 \times 10^{13}$ & 0 & 32,790 & [35] \\
\hline 68 & $\mathrm{OH}+\mathrm{H}+\mathrm{M} \rightarrow \mathrm{H}_{2} \mathrm{O}+\mathrm{M}$ & $2.20 \times 10^{22}$ & -2.0 & 0 & [35] \\
\hline 69 & $\mathrm{H}+\mathrm{H}+\mathrm{M} \rightarrow \mathrm{H}_{2}+\mathrm{M}$ & $1.80 \times 10^{18}$ & -1.0 & 0 & [35] \\
\hline
\end{tabular}

a: this work, see text; b: pre-exponential factor has been modified compared to the cited reference; c: activation energy has been modified compared to the cited reference; d: collisional coefficients $\mathrm{CH}_{3} \mathrm{OCH}_{3}: 3 \mathrm{CH}_{4}: 6.5 \mathrm{CO}: 0.75 \mathrm{CO}_{2}: 1.5 \mathrm{H}_{2}: 1 \mathrm{H}_{2} \mathrm{O}: 6.5 \mathrm{~N}_{2}: 0.4 \mathrm{O}_{2}: 0.4$; e: collisional coefficients $\mathrm{CH}_{4}: 6.5 \mathrm{CO}: 0.75 \mathrm{CO}_{2}: 1.5 \mathrm{H}_{2}: 1 \mathrm{H}_{2} \mathrm{O}: 6.5 \mathrm{~N}_{2}: 0.4 \mathrm{O}_{2}: 0.4$; f: troe: $\mathrm{A}=0.454$, T3 = 1E-30, $\mathrm{T} 1$ = 2510.0; g: troe: $\mathrm{A}=0.414$, T3 = 279.0, $\mathrm{T} 1=5459.0$.

\subsection{Base Mechanism}

The reactions in the $\mathrm{H} / \mathrm{C}_{1} / \mathrm{O}$ submechanism are the same 42 irreversible reactions (reactions 28-69 in Table 3) as in previously presented work by Larsson et al. [35], and then later by Zettervall et al. [36,41,43,49-51], but with tuning of some rate constants, as noted in Table 3 . These adjustments, applied on ten reactions, are mainly made in order to adjust the production and consumption of the radical pool $(\mathrm{O}, \mathrm{H}, \mathrm{OH})$. The submechanism originates from the 35-step methane-air mechanism, SG35, created by Smooke and Giovangigli [52] with modifications resulting in the Z42 mechanism described by Larsson et al. [35]. The base mechanism Z42 has been extensively validated in laminar cases and implemented in full combustion LES' $[35,40,42,53,54]$.

\subsection{Fuel Breakdown Reactions}

In the following, text reactions are presented with the same numbering as given in Table 3. The small size of the DME molecule enables a semi-detailed modelling of the reactions describing the fuel breakdown, with no need to incorporate global fuel breakdown reactions. In the presented mechanism fuel breakdown is initialized by either

$$
\mathrm{CH}_{3} \mathrm{OCH}_{3}+\mathrm{M} \rightarrow \mathrm{CH}_{3}+\mathrm{CH}_{3} \mathrm{O}+\mathrm{M}
$$

or

$$
\mathrm{CH}_{3} \mathrm{OCH}_{3}+\mathrm{O}_{2} \rightarrow \mathrm{CH}_{3} \mathrm{OCH}_{2}+\mathrm{HO}_{2} \text {, }
$$

with the thermal decomposition reaction R1 being more closely associated with high temperature oxidation and the oxygen oxidation reaction $\mathrm{R} 2$ being more active at lower temperatures, both of which are general hydrocarbon oxidation features [55]. H-abstraction from $\mathrm{CH}_{3} \mathrm{OCH}_{3}$ by any of the radicals $\mathrm{H}$, $\mathrm{O}$ or $\mathrm{OH}$ (R2-R4) actualizes as soon as a sufficient radical pool has been formed. $\mathrm{H}$-abstraction from the fuel can also occur via intermediate oxygenated hydrocarbon $\left(\mathrm{CH}_{3} \mathrm{O}_{2}, \mathrm{CH}_{3} \mathrm{CO}\right.$ and $\mathrm{CH}_{3} \mathrm{O}$, R5-R7) or oxygen molecules. Reaction $\mathrm{R} 1$ is a direct link between the fuel breakdown and the underlying $\mathrm{H} / \mathrm{C}_{1} / \mathrm{O}$ base 
mechanism. Except from reaction R1, all fuel breakdown reactions are H-abstraction reactions, creating $\mathrm{CH}_{3} \mathrm{OCH}_{2}$, which is then oxidized further in the intermediate hydrocarbon submechanism.

\subsection{Intermediate Hydrocarbon Oxidation}

The reactions presented in this submechanism represent a bridge between the submechaisms "fuel breakdown" and "base mechanism". The reactions in this submechanism are adopted from the mechanisms by Burke et al. [17], Fischer et al. [28] and Khare et al. [32]. The reactions are chosen to represent a minimum set of reactions without compromising the completeness of the chemical representation, i.e., all important intermediate compounds are included but their main reaction channels are chosen while minor reaction channels are omitted. Once the $\mathrm{CH}_{3} \mathrm{OCH}_{2}$ has been formed through $\mathrm{H}$-abstraction of the fuel, it then oxidizes through

$$
\mathrm{CH}_{3} \mathrm{OCH}_{2} \rightarrow \mathrm{CH}_{2} \mathrm{O}+\mathrm{CH}_{3}
$$

or

$$
\mathrm{CH}_{3} \mathrm{OCH}_{2}+\mathrm{O}_{2} \rightarrow \mathrm{CH}_{3} \mathrm{OCH}_{2} \mathrm{O}_{2} \text {. }
$$

Reactions R10 and R11 are both involved in the oxidation at lower temperatures [17]. Reaction R10 creates (in the same way as R1) a bridge to the base mechanism, bypassing the low-temperature kinetics. Reaction R11 on the other hand initializes one low- and medium-temperature kinetic pathway with the product $\mathrm{CH}_{3} \mathrm{OCH}_{2} \mathrm{O}_{2}$ proceeding through reactions $\mathrm{R} 12-\mathrm{R} 16$.

$$
\mathrm{CH}_{3} \mathrm{OCH}_{2} \mathrm{O}_{2}+\mathrm{CH}_{3} \mathrm{OCH}_{2} \mathrm{O}_{2} \rightarrow \mathrm{O}_{2}+\mathrm{CH}_{3} \mathrm{OCH}_{2} \mathrm{O}+\mathrm{CH}_{3} \mathrm{OCH}_{2} \mathrm{O}
$$

and

$$
\mathrm{CH}_{3} \mathrm{OCH}_{2} \mathrm{O}_{2}+\mathrm{CH}_{3} \mathrm{CHO} \rightarrow \mathrm{CH}_{3} \mathrm{OCH}_{2} \mathrm{O}_{2} \mathrm{H}+\mathrm{CH}_{3} \mathrm{CO}
$$

both result in $\mathrm{CH}_{3} \mathrm{OCH}_{2} \mathrm{O}$, either directly (R12) or via

$$
\mathrm{CH}_{3} \mathrm{OCH}_{2} \mathrm{O}_{2} \mathrm{H} \rightarrow \mathrm{CH}_{3} \mathrm{OCH}_{2} \mathrm{O}+\mathrm{OH} \text {. }
$$

$\mathrm{CH}_{3} \mathrm{OCH}_{2} \mathrm{O}$ continues the oxidation pathway through

$$
\mathrm{CH}_{3} \mathrm{OCH}_{2} \mathrm{O} \rightarrow \mathrm{CH}_{3} \mathrm{O}+\mathrm{CH}_{2} \mathrm{O}
$$

which is essential for the oxidation process at low and medium temperatures. A second route for $\mathrm{CH}_{3} \mathrm{OCH}_{2} \mathrm{O}_{2}$ is also provided, where it is reconfigured to $\mathrm{CH}_{2} \mathrm{OCH}_{2} \mathrm{O}_{2} \mathrm{H}$. $\mathrm{CH}_{2} \mathrm{OCH}_{2} \mathrm{O}_{2} \mathrm{H}$ can then decompose as

$$
\mathrm{CH}_{2} \mathrm{OCH}_{2} \mathrm{O}_{2} \mathrm{H} \rightarrow \mathrm{CH}_{2} \mathrm{O}+\mathrm{CH}_{2} \mathrm{O}+\mathrm{OH}
$$

or react with oxygen molecules,

$$
\mathrm{CH}_{2} \mathrm{OCH}_{2} \mathrm{O}_{2} \mathrm{H}+\mathrm{O}_{2} \rightarrow \mathrm{HO}_{2} \mathrm{CH}_{2} \mathrm{OCHO}+\mathrm{OH}
$$

$\mathrm{HO}_{2} \mathrm{CH}_{2} \mathrm{OCHO}$ can thermally decompose to $\mathrm{CH}_{2} \mathrm{O}, \mathrm{CO}_{2}, \mathrm{OH}$ and $\mathrm{H}$ via reaction $\mathrm{R} 20$ or to $\mathrm{CH}_{2} \mathrm{O}, \mathrm{CO}$ and two $\mathrm{OH}$ via reaction $\mathrm{R} 21$. Both of these reactions, forming $\mathrm{C}_{1}$ species and fast radicals, are important for the ignition at low temperatures. Finally, reactions

$$
\begin{aligned}
\mathrm{CH}_{3}+\mathrm{O}_{2}+\mathrm{M} & \rightarrow \mathrm{CH}_{3} \mathrm{O}_{2}+\mathrm{M}, \quad \text { (R27) } \\
\mathrm{CH}_{3} \mathrm{O}_{2}+\mathrm{CH}_{3} \mathrm{O}_{2} & \rightarrow \mathrm{O}_{2}+\mathrm{CH}_{3} \mathrm{O}+\mathrm{CH}_{3} \mathrm{O}, \quad \text { (R25) } \\
\mathrm{CH}_{3} \mathrm{OCH}_{2} \mathrm{O}_{2}+\mathrm{CH}_{3} \mathrm{OCH}_{3} & \rightarrow \mathrm{CH}_{3} \mathrm{OCH}_{2} \mathrm{O}_{2} \mathrm{H}+\mathrm{CH}_{3} \mathrm{CO}+\mathrm{H}_{2}
\end{aligned}
$$

and

$$
\mathrm{CH}_{3} \mathrm{OH}+\mathrm{M} \rightarrow \mathrm{CH}_{3}+\mathrm{OH}+\mathrm{M}
$$


produce and/or oxidize the intermediate oxygenated species $\mathrm{CH}_{3} \mathrm{O}_{2}, \mathrm{CH}_{3} \mathrm{CO}$ and $\mathrm{CH}_{3} \mathrm{OH}$.

\section{Modelling Details}

Modelling of the laminar flame propagation, ignition delay times, extinction strain rate and sensitivity analysis were performed in $0 \mathrm{D}$ and $1 \mathrm{D}$ reactors using the Cantera software 2.3.0 [56]. Adaptive grid refinements were used for the laminar propagation simulations, resulting in grids using roughly 650 grid points for all three mechanisms. Ignition delay times were simulated using constant pressure assumptions, with time steps of $1.0 \times 10^{-7}$. The extinction strain rate, $\sigma_{\text {ext, }}$ is simulated using a non-premixed model set-up with a stream of fuel meeting a stream of air resulting in a counterflow flame at $\mathrm{T}=300 \mathrm{~K}$ and pressures of 1, 3 and $10 \mathrm{~atm}$. Thermodynamic data were adopted from the databases provided by Burcat et al. [57], Fischer et al. [28], Burke et al. [17], and Khare et al. [32], and transport data from Fischer et al. [28] and Khare et al. [32].

\section{Mechanism Validation}

The mechanism validation targets are the laminar burning velocity, flame temperature, major species concentrations $\left(\mathrm{CO}, \mathrm{CO}_{2}, \mathrm{H}_{2} \mathrm{O}, \mathrm{H}_{2}\right)$, key minor species $\left(\mathrm{CH}_{3} \mathrm{O}, \mathrm{CH}_{2} \mathrm{O}, \mathrm{OH}\right.$, $\mathrm{O})$, ignition delay times and extinction strain rates. Regarding the species chosen, $\mathrm{CH}_{3} \mathrm{O}$ represents a key intermediate hydrocarbon and a product species from fuel breakdown, reaction $\mathrm{R} 1$, as well as a product species from the thermal decomposition of $\mathrm{CH}_{3} \mathrm{OCH}_{2} \mathrm{O}$, reaction $\mathrm{R} 23$. $\mathrm{CH}_{2} \mathrm{O}$ is a key intermediate hydrocarbon being present throughout the intermediate hydrocarbon oxidation and base submechanisms, and $\mathrm{OH}$ and $\mathrm{O}$ are key radicals in all hydrocarbon combustion. The laminar burning velocities were simulated for a wide range of initial gas pressures, $p=1 \mathrm{~atm}$ to $10 \mathrm{~atm}$, and temperatures, $\mathrm{T}=300 \mathrm{~K}$ to $750 \mathrm{~K}$, and for fuel lean to fuel rich conditions, $\phi=0.5$ to $\phi=1.8$. For the ignition delay time the range in equivalence ratios goes from fuel lean to fuel rich, $\phi=0.5$ to $\phi=2.0$, with pressures from $p=2$ to $25 \mathrm{~atm}$. The pressure range for the extinction strain rate simulations goes from $p=1 \mathrm{~atm}$ to $10 \mathrm{~atm}$, at an initial temperature of $300 \mathrm{~K}$.

The detailed reaction mechanisms by Fischer et al. [28], Burke et al. [17] and the San Diego mechanism [30] and the reduced reaction mechanisms by Pan et al. [33] and Khare et al. [32] are used for comparison. Experimental data is included where possible, and all experimental data and a full range of simulation parameters are listed in Table 2.

\subsection{Laminar Burning Velocity}

Figure 2 shows simulation results for laminar burning velocity at standard conditions, defined here to be $1 \mathrm{~atm}$ and $300 \mathrm{~K}$. For all equivalence ratios investigated, Z69 is in good agreement with the experimental data and the detailed reaction mechanisms. The spread in laminar burning velocity predictions for the reference mechanisms and experimental data sets are however relatively wide, spanning up to $9 \mathrm{~cm} / \mathrm{s}$ for mechanism predictions at around stoichiometric conditions and $8 \mathrm{~cm} / \mathrm{s}$ for experimental results at highly fuel rich conditions. These differences are large when comparing to other more well-characterised alkane and alkene fuels. The reduced mechanism by Pan et al. [33] completely fails at predicting the laminar burning velocity, and the fact that that reaction mechanism was not designed to model laminar burning velocities is clearly shown in the results below. The main focus of that mechanism is to predict ignition delay times, and since the aim of the present work is to present reaction mechanisms capable of predicting a range of flame parameters, and due to the poor performance of the reaction mechanism by Pan et al. [33] for laminar burning velocities, it will not be included in further comparisons. 


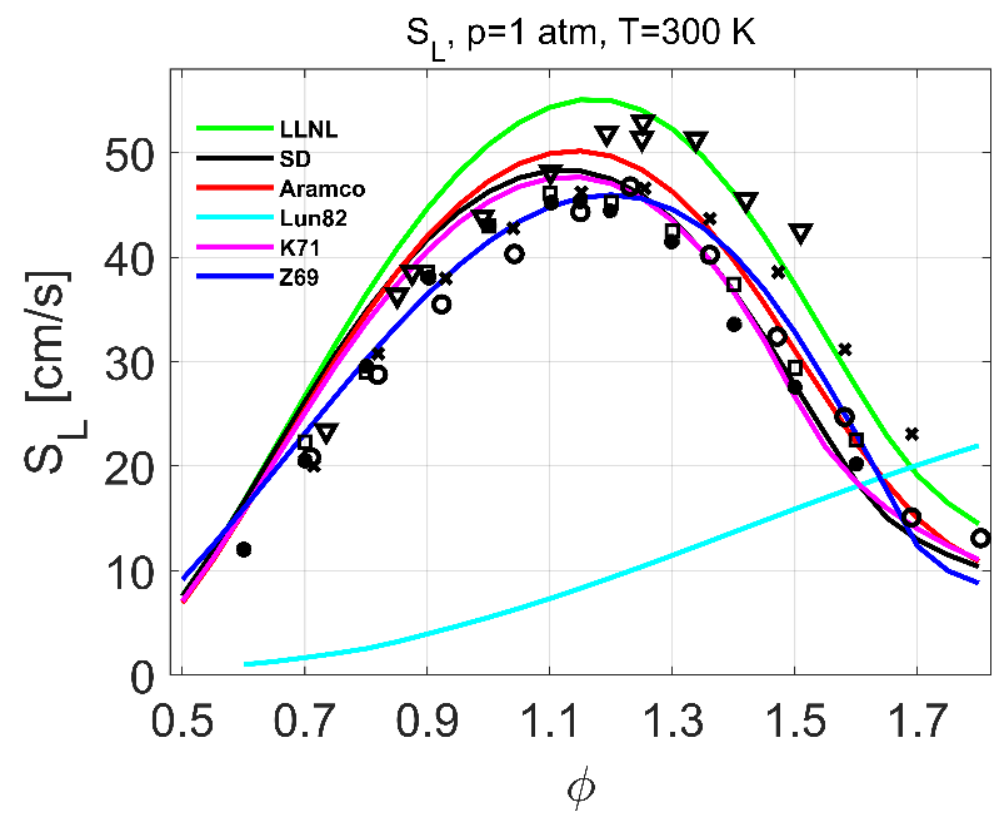

Figure 2. Laminar burning velocities for DME-air mixtures versus equivalence ratio, simulated at $1 \mathrm{~atm}$ and $300 \mathrm{~K}$. Legend: experimental data for $S_{\mathrm{L}}$ from $(\bigcirc)$ Daly et al. [21], ( $\times$ ) Qin et al. [23], ( $\left.\square\right)$ Vries et al. [26], (•) Wang et al. [25], ( $\mathbf{\nabla})$ Zhao et al. [22]. Mechanism predictions from Z69 in blue, LLNL in green [28], Aramco in red [17], K71 in magenta [32], San Diego in black [30] and Pan82 in cyan [33].

Sensitivity analysis identifies the reactions with the largest importance for a particular flame parameter. Figure $3 a-e$ present the results for the fourteen most sensitive reactions in each reaction mechanism for the laminar burning velocity, at stoichiometric conditions standard and $1 \mathrm{~atm}$ and $300 \mathrm{~K}$. Figure 3a-e clearly show that reactions in the $\mathrm{H} / \mathrm{C}_{1} / \mathrm{O}$ chemistry dominate the modelling of the laminar burning velocity in all reaction mechanisms. The reactions with the highest positive sensitivities in the detailed mechanisms are the usual suspects: $\mathrm{H}+\mathrm{O}_{2} \rightarrow \mathrm{O}+\mathrm{OH}, \mathrm{CO}+\mathrm{OH} \rightarrow \mathrm{CO}_{2}+\mathrm{H}$ and $\mathrm{HCO}+\mathrm{M} \rightarrow \mathrm{CO}+\mathrm{H}+\mathrm{M}$, and the reactions with the highest negative sensitivities are generally chain terminating or $\mathrm{H}$-consuming reactions. The importance of the chain branching and chain terminating reactions determining the laminar burning velocity is a common feature for all hydrocarbon and oxygenated hydrocarbon fuels. Few reactions involved in the breakdown of the fuel or oxidation of intermediate fuel products show high importance for the prediction of the laminar burning velocity.

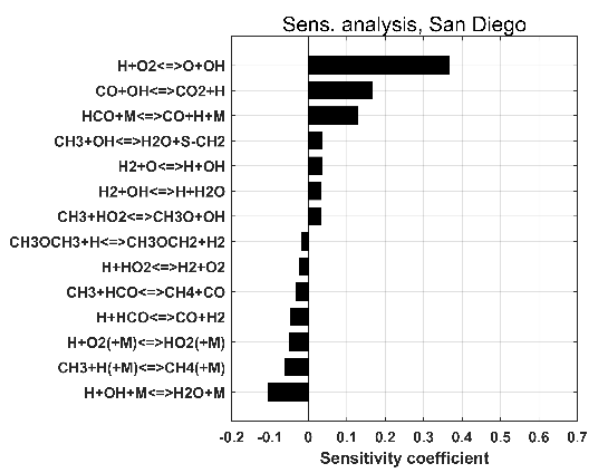

(a)

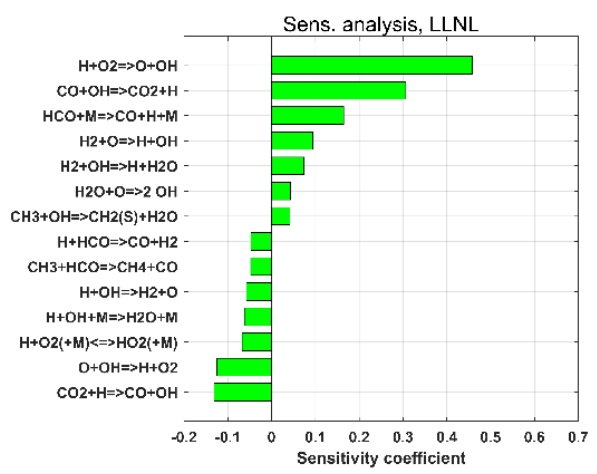

(b)

Figure 3. Cont. 


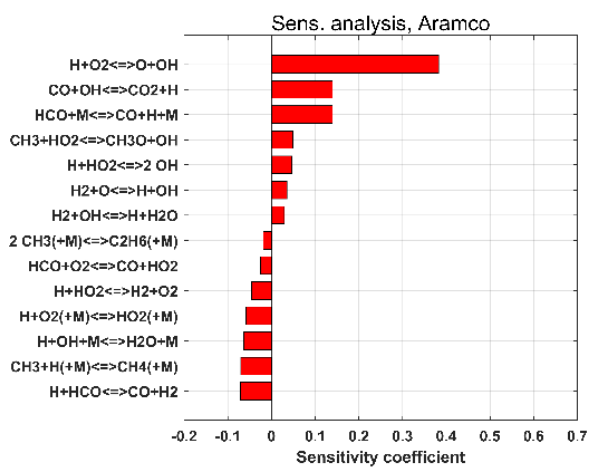

(c)

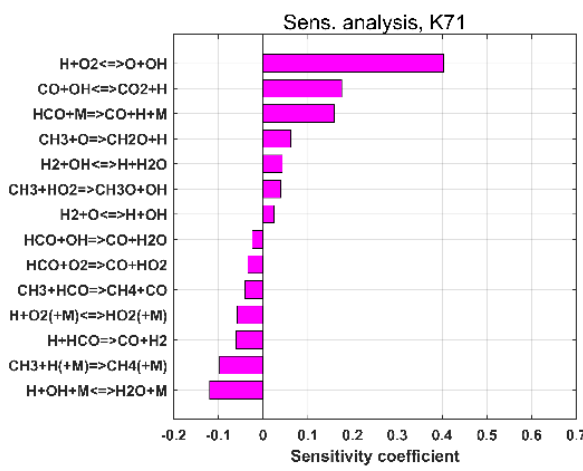

(e)

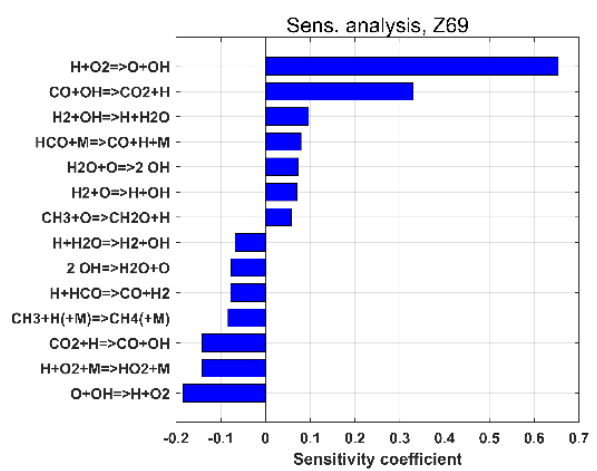

(d)

Figure 3. Sensitivity spectra of the fourteen most sensitive reactions for the San Diego mechanism in (a), the LLNL mechanism in (b), the Aramco mechanism in (c), Z69 in (d) and K71 in (e), all at $\phi=1.0,1 \mathrm{~atm}$ and $300 \mathrm{~K}$.

Figure 4 shows the effect of increasing initial gas pressure, Figure $4 a$, and temperature, Figure $4 b$, on the laminar burning velocity. All five reaction mechanisms react similarly when increasing the pressure to either 5 or $10 \mathrm{~atm}$, resulting in lower laminar burning velocities. The K71 mechanism however has a larger decrease in laminar burning velocity as the pressure increases, slightly underpredicting the velocity. Both Z69 and the detailed reaction mechanisms match the experimental data reasonably well. The relative relation between the mechanisms at $1 \mathrm{~atm}$ and $300 \mathrm{~K}$ holds when the initial gas temperature is increased to $450 \mathrm{~K}, 600 \mathrm{~K}$ and $750 \mathrm{~K}$ at $1 \mathrm{~atm}$. Note that the simulation using the Aramco mechanism did not converge to provide a solution at the highest temperature.

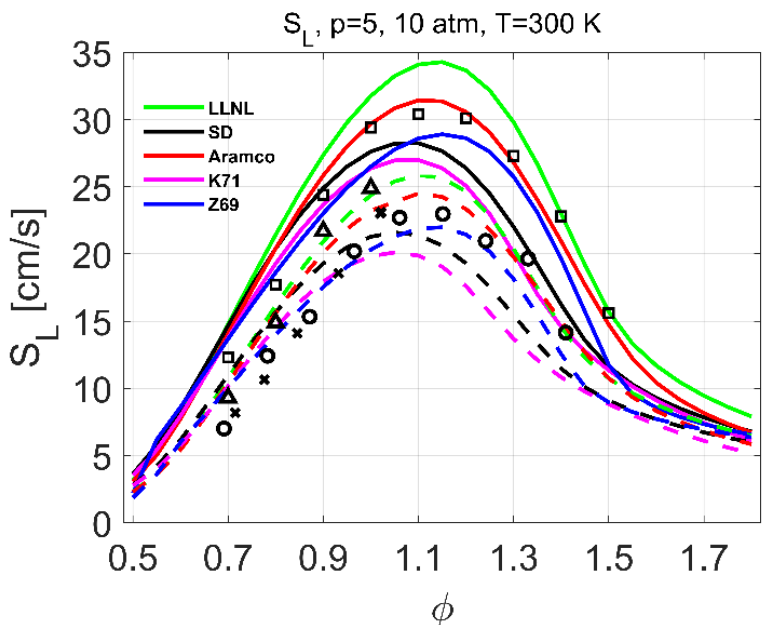

(a)

Figure 4. Cont. 


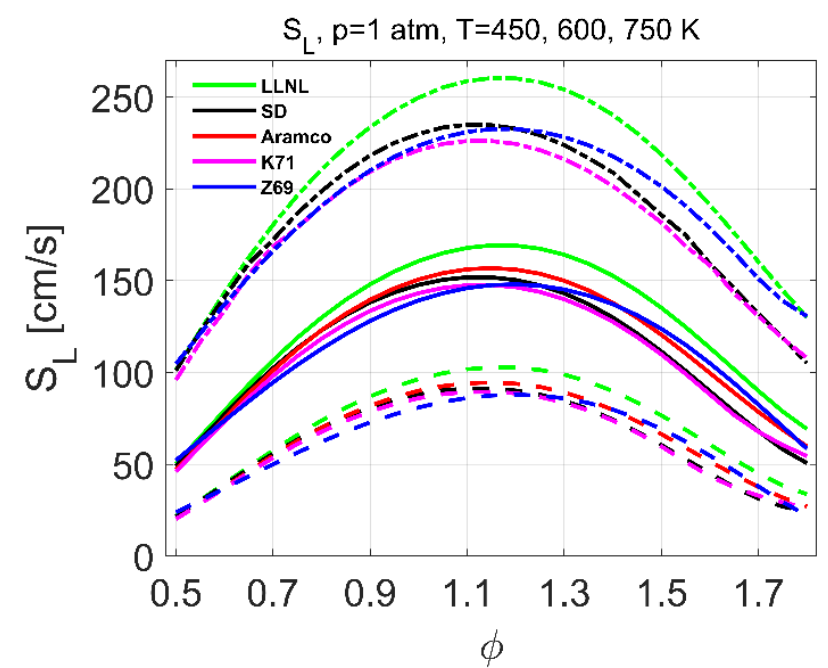

(b)

Figure 4. Laminar burning velocities for DME-air mixtures with varying pressures of $5 \mathrm{~atm}$ (solid line) and $10 \mathrm{~atm}$ (dashed line) in (a) and temperatures of $450 \mathrm{~K}$ (dashed line), $600 \mathrm{~K}$ (solid line) and $750 \mathrm{~K}$ (dot-dashed line) in (b). Legend: experimental data for SL from ( $\times$ ) Qin et al. [23] at 10 atm, $(\square)$ and (ム) Vries et al. [26] at 5 and $10 \mathrm{~atm}$, respectively, and ( $\bigcirc)$ Jomaas et al. [24] at $10 \mathrm{~atm}$. Mechanism predictions from Z69 in blue, LLNL in green [28], Aramco in red [17], K71 in magenta [32] and San Diego in black [30].

\subsection{Ignition Delay Time}

The second development target is the ignition delay time, with the specific target conditions summarized in Table 2. The definition of when ignition has occurred is when the temperature has increased by $300 \mathrm{~K}$, matching the rise in pressure and the peak of $\mathrm{OH}^{*}$ as explained by Zettervall et al. [49]. The complex low- and medium-temperature ignition characteristics of DME compared to short chained alkanes and alkenes mean that even reduced reaction mechanisms must include a considerable number of reactions for describing the low- and medium-temperature process. The intermediate hydrocarbon oxidation submechanism in Z69 incorporates some of the main reaction pathways of the low- and medium-temperature reaction kinetics in order to capture the NTC behaviour. The hightemperature reaction kinetics is mainly present in the fuel breakdown submechanism and in the $\mathrm{H} / \mathrm{C}_{1} / \mathrm{O}$ kinetics in the base mechanism. The performance of the base mechanism on high-temperature ignition delay times has been tested for a range of fuels [35,43,49-51]. Additional figures for 11 and $25 \mathrm{~atm}$ are shown in Appendix A.

Ignition delay times for all five reaction mechanisms and experimental data at a pressure of $25 \mathrm{~atm}$ and $\phi=0.5$ and $\phi=1.0$ are shown in Figure 5. Here, Z69 is the only mechanism matching the experimental data at temperatures below $900 \mathrm{~K}$ and $\phi=0.5$, Figure $5 \mathrm{a}$. The ignition delay times predicted by the mechanisms converge when the temperature increases, matching the experimental data. At lower temperatures all reference mechanisms taper off with only small NTC behaviours, where the NTC behaviour predicted by Z69 is more pronounced. The highly reduced set of reactions in Z69 compared to the other, larger mechanisms results in a simpler description of reaction pathways present, especially during the ignition process. Since the majority of the pathways for fuel lean conditions are kept, and the reduction focuses more on reactions modelling the fuel rich conditions, the modelling accuracy is higher at fuel lean conditions. This fact is seen when comparing the NTC behaviour for Z69, which is more accentuated at fuel rich conditions (see Appendix A). All mechanisms show a slight decrease in ignition with an increase in equivalence ratio, as is also shown by Burke et al. [17]. 


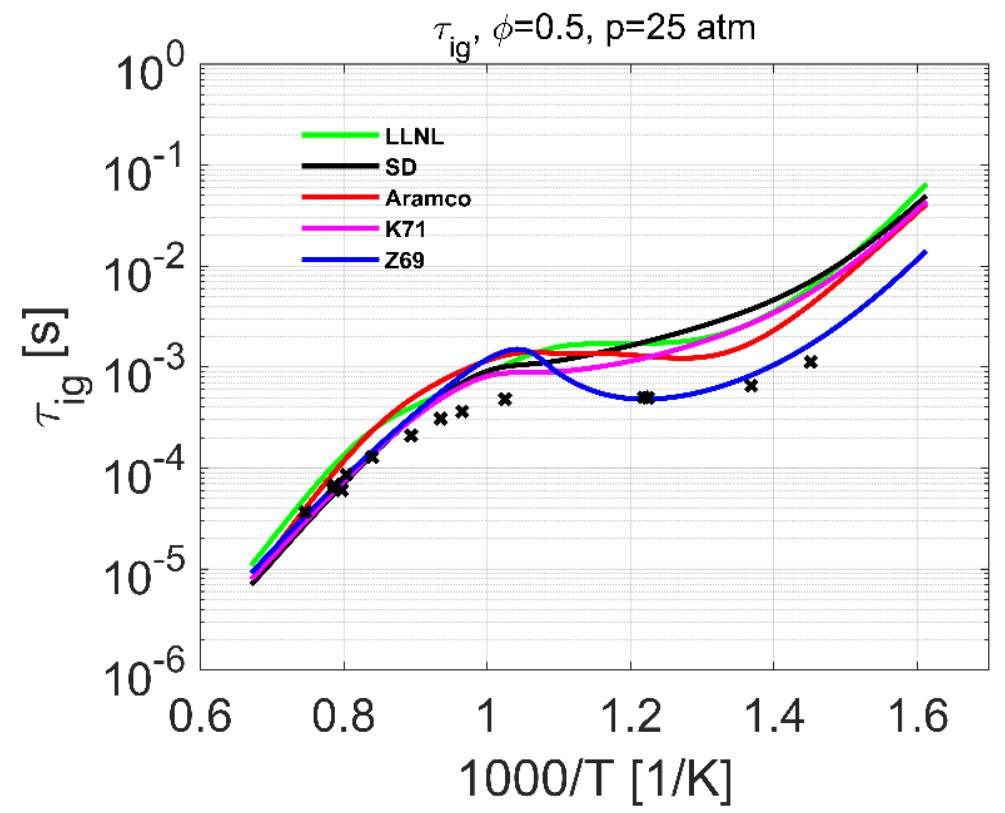

(a)

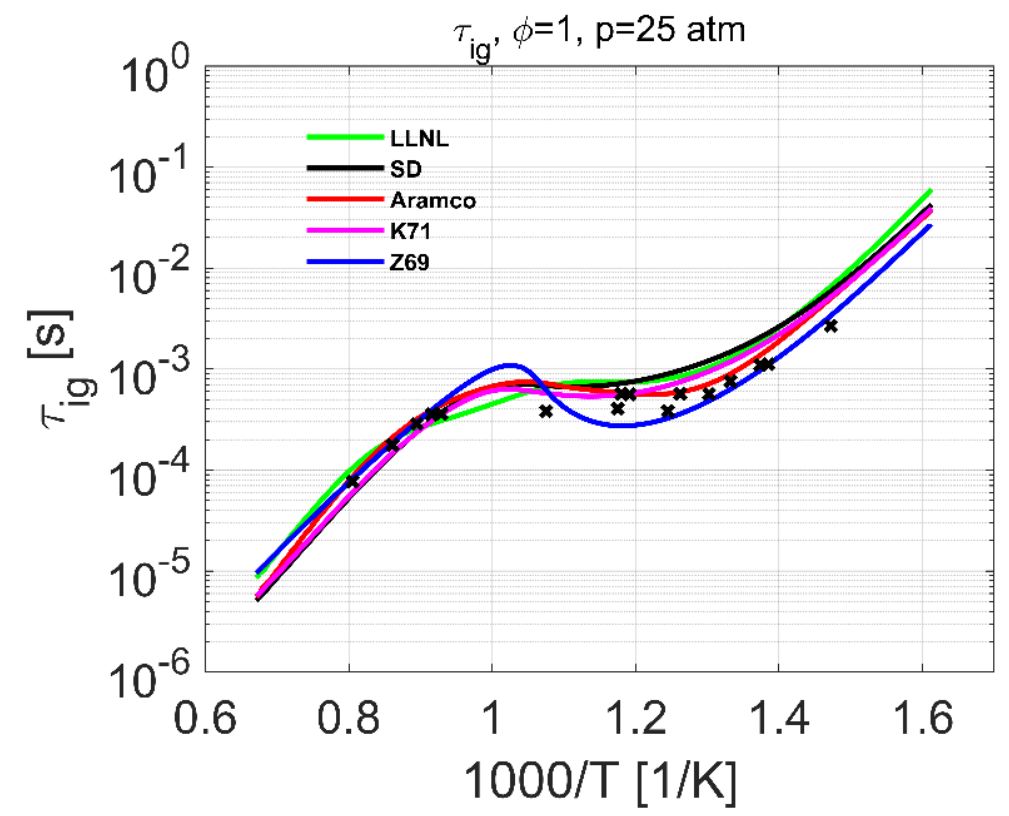

(b)

Figure 5. Ignition delay time for DME-air mixtures at $25 \mathrm{~atm}$ and $\phi=0.5 \mathrm{in}$ (a) and $\phi=1.0$ in (b). Legend: experimental data for $\tau$ ig from $(\times)$ Burke et al. [17]. Mechanism predictions from Z69 in blue, LLNL in green [28], Aramco in red [17], K71 in magenta [32] and San Diego in black [30].

Figure 6 show the ignition delay times at $10 \mathrm{~atm}$ and $\phi=0.5$ in (a) and $\phi=1.0$ in (b), respectively. As for the higher pressure in Figure 5, all mechanism predictions converge to similar ignition delay times at temperatures above $1000 \mathrm{~K}$, indicating strong similarities in their respective high temperature kinetics. Z69 again show a stronger NTC behaviour than the other mechanisms, with a reasonable agreement to the experimental data. K71 has a less pronounced NTC curve compared to Z69, whereas the NTC behaviour of the detailed mechanisms goes from none to relatively strong, depending on the equivalence ratio. The spread in ignition delay times between the mechanisms is greater than at $2 \mathrm{~atm}$ in Figure 7. 


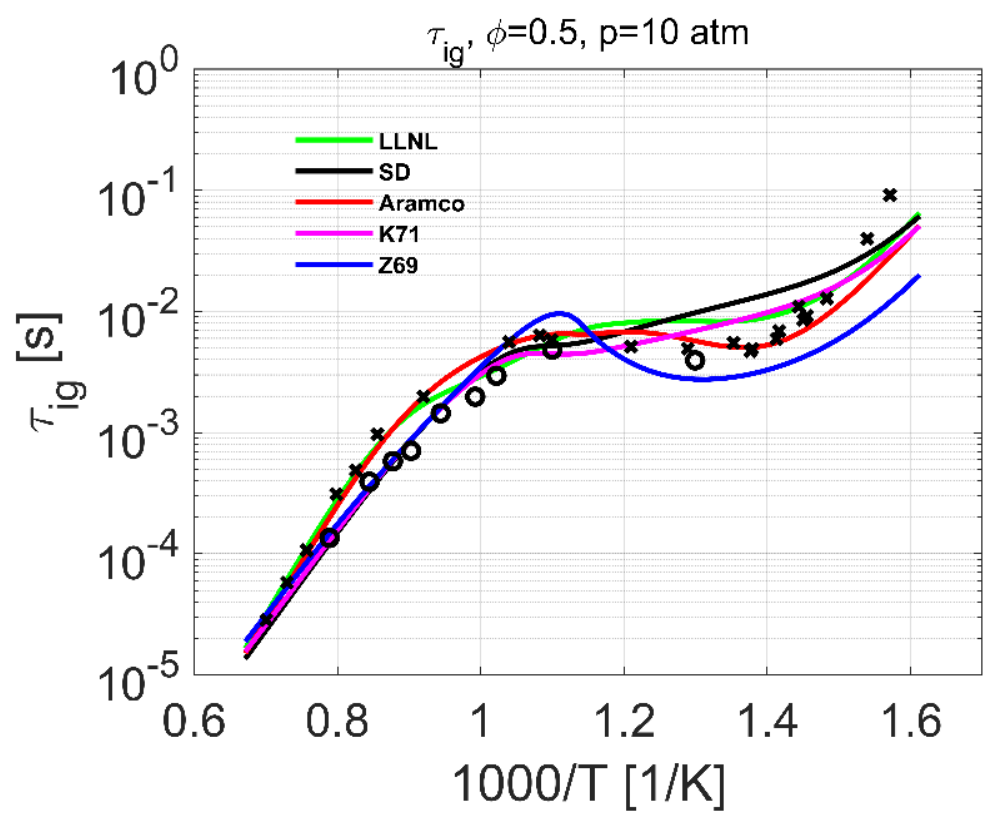

(a)

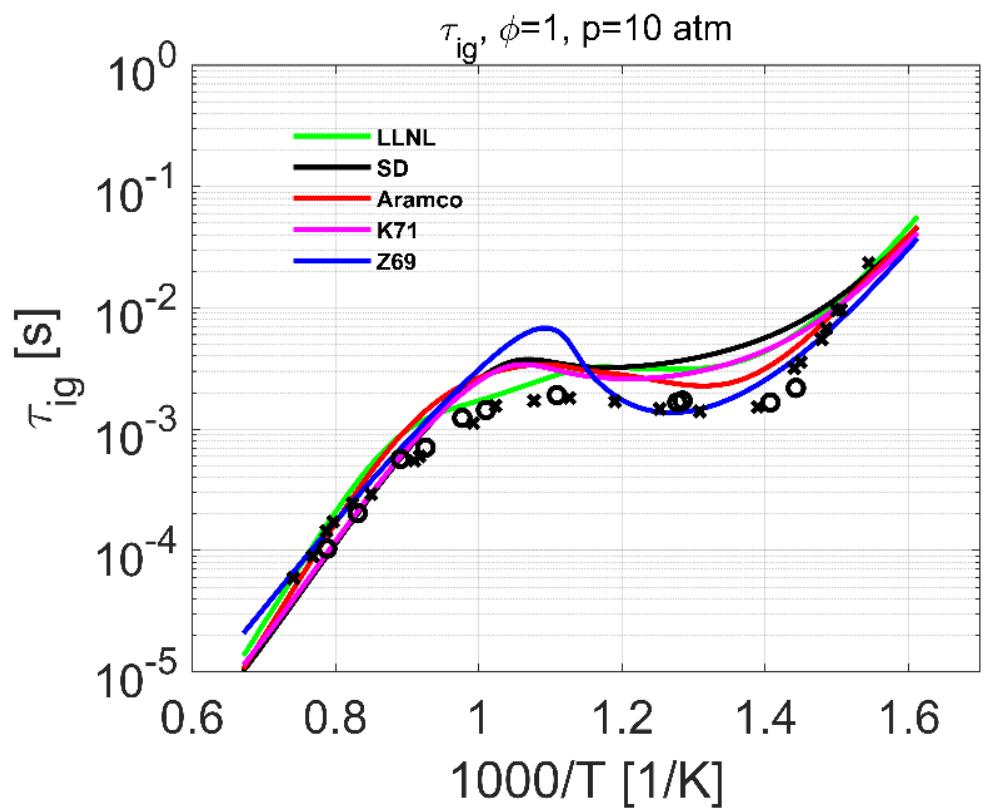

(b)

Figure 6. Ignition delay time for DME-air mixtures at $10 \mathrm{~atm}$ and $\phi=0.5$ in (a) and $\phi=1.0$ in (b). Legend: experimental data for $\tau$ ig from $(\bigcirc)$ Jiang et al. [15] and $(\times)$ Burke et al. [17]. Mechanism predictions from Z69 in blue, LLNL in green [28], Aramco in red [17], K71 in magenta [32] and San Diego in black [30].

Ignition delay times at $2 \mathrm{~atm}$ and $\phi=0.5$ and $\phi=1.0$ are shown in Figure $7 \mathrm{a}, \mathrm{b}$, respectively. As for the higher pressures of 10 and $25 \mathrm{~atm}$, the predicted ignition delay times converge when the temperature increases, matching the experimental data.

The ignition predicted by Z69 displays a stronger NTC behaviour, at all pressures, than the other mechanisms. This is often due to a peak in ignition time estimate at around $1000 \mathrm{~K}$ and it can be seen for all pressures investigated. 


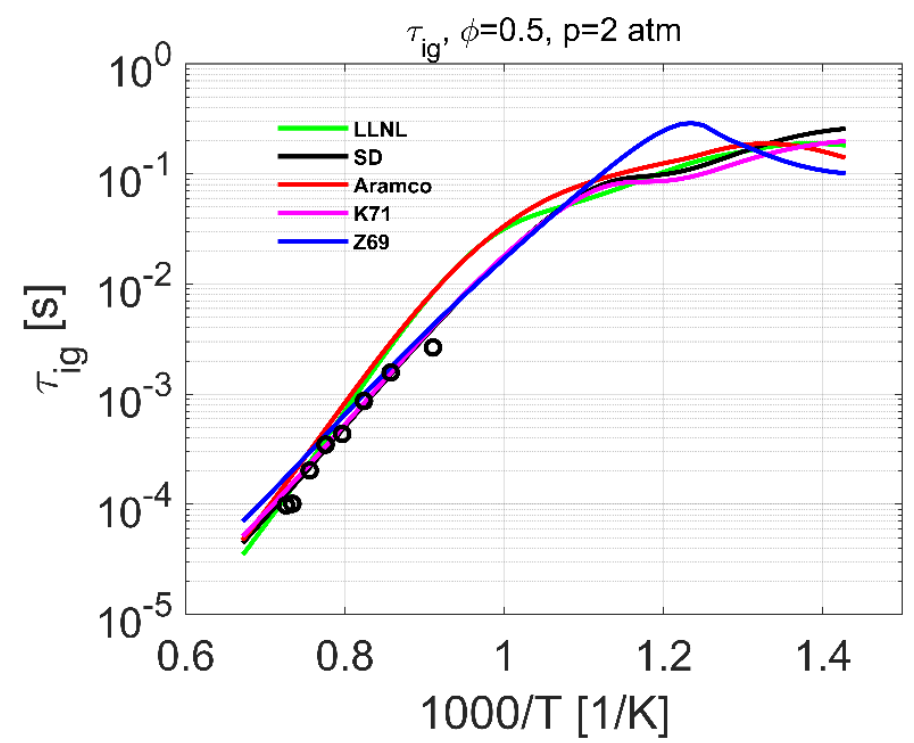

(a)

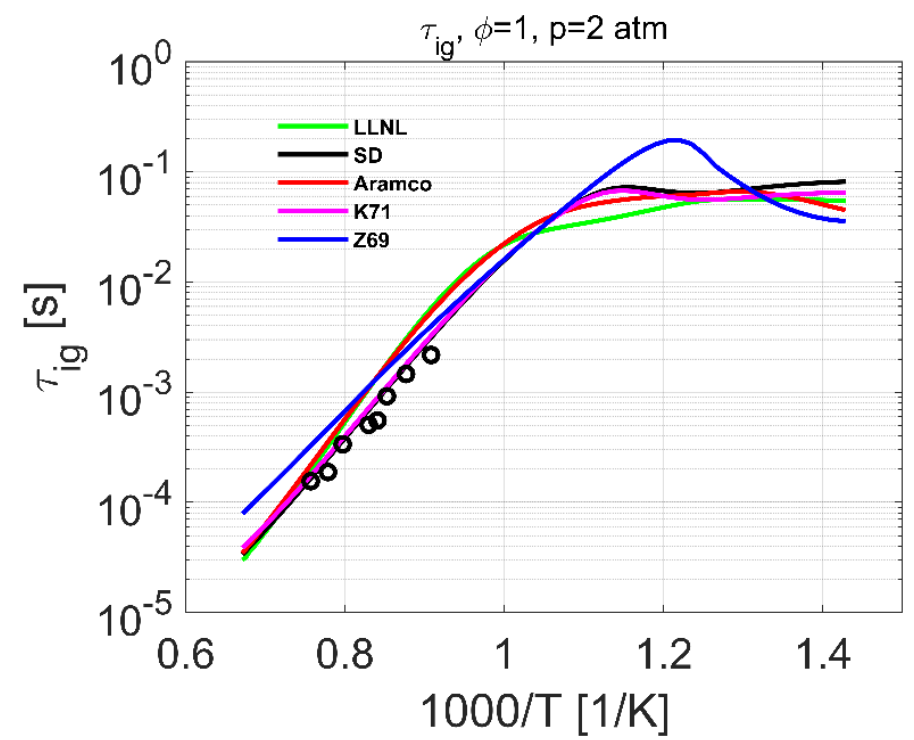

(b)

Figure 7. Ignition delay time for DME-air mixtures at 2 atm and $\phi=0.5$ in (a) and $\phi=1.0$ in (b). Legend: experimental data for $\tau$ ig from $(\bigcirc)$ Jiang et al. [15]. Mechanism predictions from Z69 in blue, LLNL in green [28], Aramco in red [17], K71 in magenta [32] and San Diego in black [30].

\subsection{Extinction Strain Rate}

The extinction strain rate determines at what extinction limit the flame will no longer propagate. This means that a too high predicted extinction limit enables a flame to burn at turbulence levels where it would otherwise be extinguished. The simulations model a case with a non-premixed condition, where a stream of fuel meets a stream of air resulting in a counterflow diffusion flame, with temperatures of $\mathrm{T}=300 \mathrm{~K}$ and pressures of 1,3 and $10 \mathrm{~atm}$. Figure 8 shows the extinction strain rate for the five reaction mechanisms at the three investigated different pressures. All reaction mechanisms show similar strain rate behaviour, with Z69 predicting the lowest strain rate and LLNL the highest. More importantly is the pressure dependence of the strain rate resulting in a significantly higher strain rate already at moderately elevated pressures, indicating that even modest pressure significantly increases the flame's ability to withstand high levels of turbulence. All mechanisms capture this shift in extinction strain rate that comes with increasing pressure. 


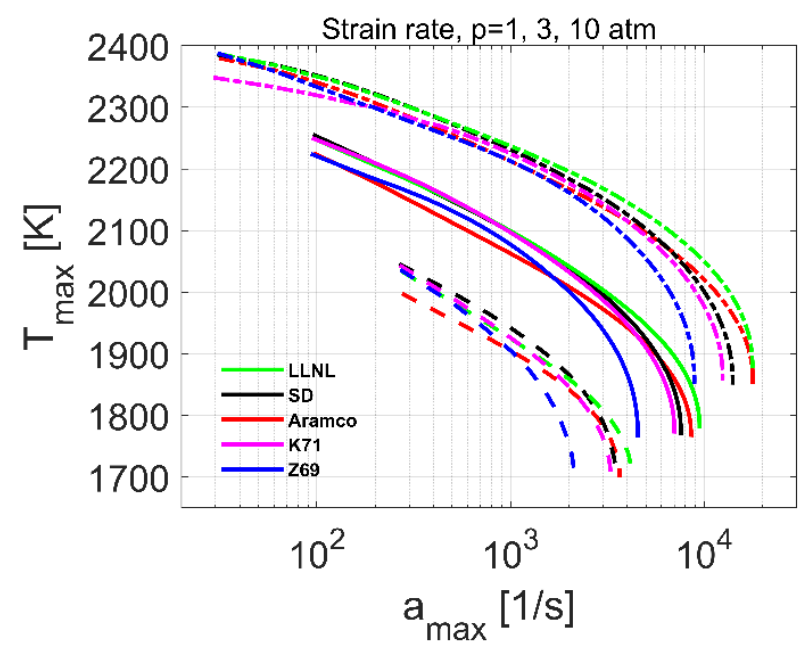

Figure 8. Extinction strain rates for DME-air mixtures at $1 \mathrm{~atm}$, solid line, $3 \mathrm{~atm}$, dashed line, and 10 atm, dot-dashed line. Legend: Mechanism predictions from Z69 in blue, LLNL in green [28], Aramco in red [17], K71 in magenta [32] and San Diego in black [30].

\subsection{Flame Profiles}

Figure 9 presents flame profiles of major species $\left(\mathrm{CO}, \mathrm{CO}_{2}\right)$ and temperature profiles, for equivalence ratios $0.6,1.0$ and 1.4. The chosen equivalence ratios represent fuel lean, stoichiometric and fuel rich conditions, all simulated at $1 \mathrm{~atm}$ and $300 \mathrm{~K}$. Flame profiles showing other major species $\left(\mathrm{H}_{2} \mathrm{O}, \mathrm{H}_{2}\right)$ and key minor species $\left(\mathrm{CH}_{3} \mathrm{O}, \mathrm{CH}_{2} \mathrm{O}, \mathrm{OH}, \mathrm{O}\right)$ are shown in Appendix A. For $\phi=0.6$ in Figure 9 a all reaction mechanisms show similar results, with $\mathrm{CO}_{2}$ reaching its maximum value shortly after the flame front. $\mathrm{CO}$ peaks in the flame front and is then oxidized further to $\mathrm{CO}_{2}$ due to the excess $\mathrm{O}_{2}$ at these operating conditions.

The stoichiometric mixture in Figure $9 \mathrm{~b}$ again shows strong similarities between all reaction mechanisms, for both $\mathrm{CO}$ and $\mathrm{CO}_{2}$, and temperature. The higher fuel-to-air ratio in Figure $9 \mathrm{c}$ means that not all $\mathrm{CO}$ is being converted into $\mathrm{CO}_{2}$ resulting in considerably higher values of $\mathrm{CO}$, the $\mathrm{CO}$ concentrations at the end of the domain being higher than $\mathrm{CO}_{2}$. All five mechanisms produce similar results regardless of species and equivalence ratios. This is also true for the other major and minor species targeted by the Z69 mechanism, with plots showing results for all five mechanisms present in Appendix A.

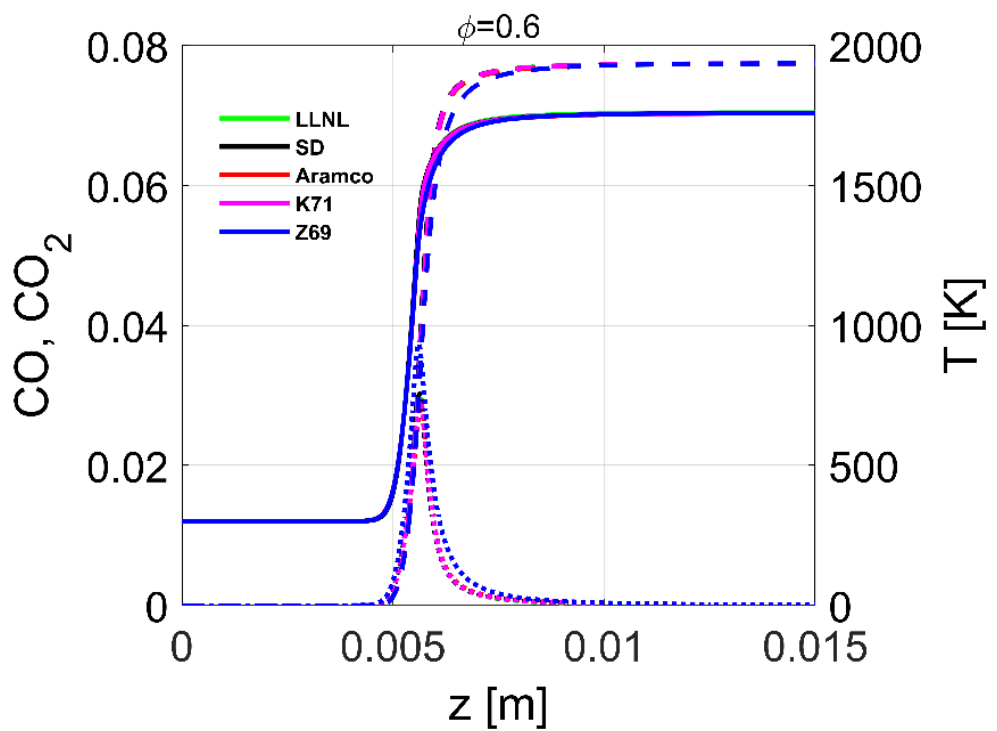

(a)

Figure 9. Cont. 


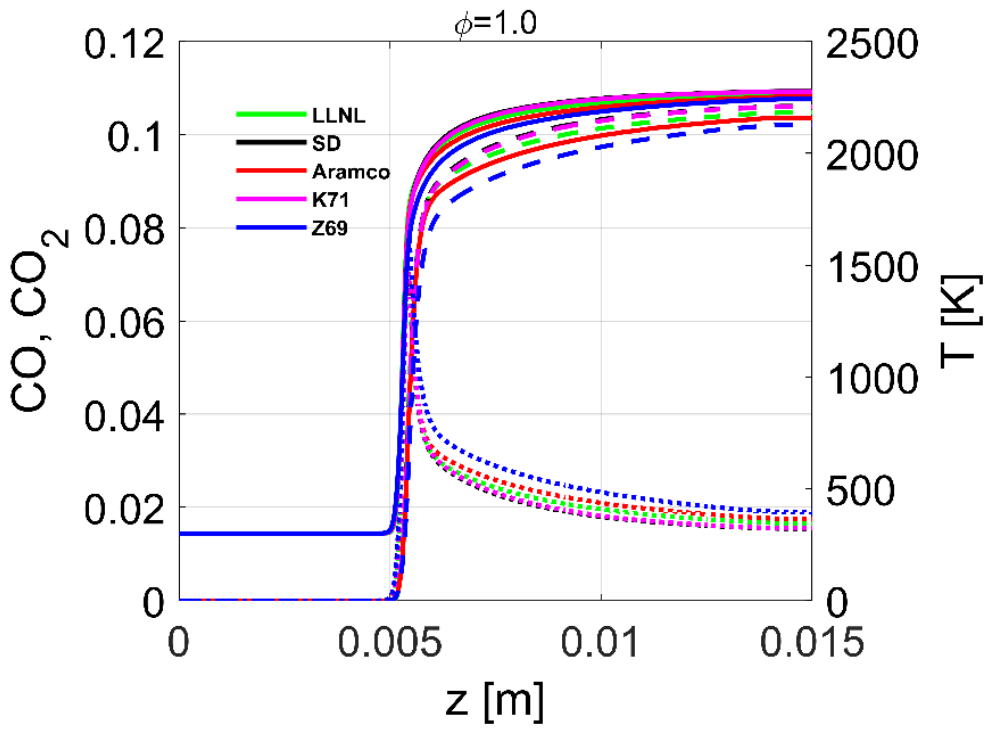

(b)

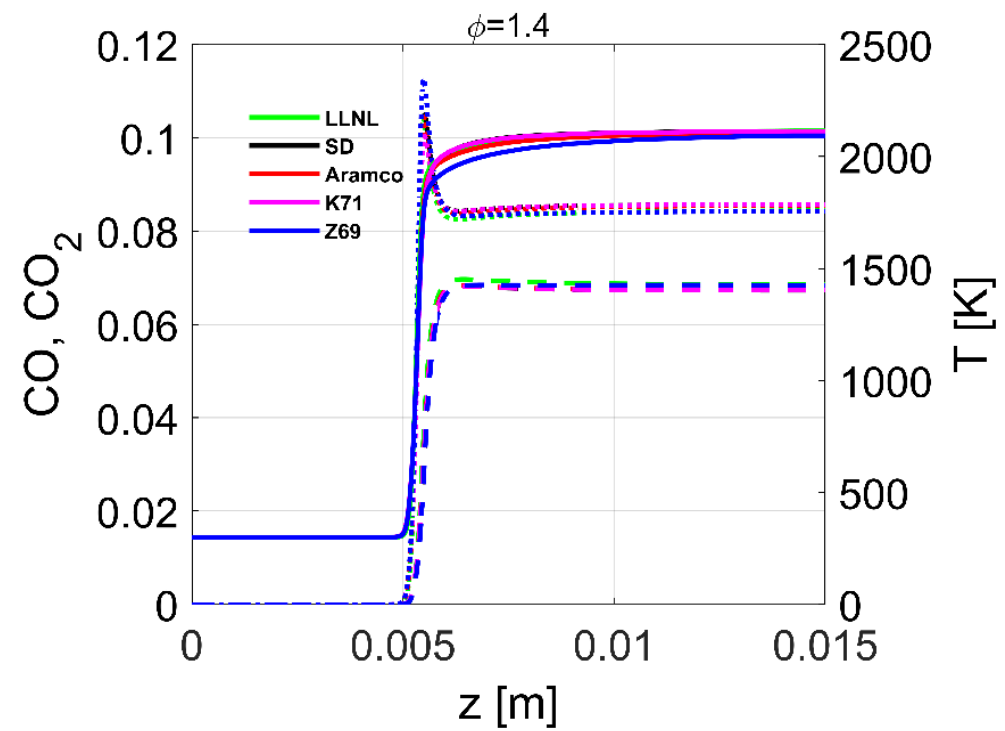

(c)

Figure 9. Flame profiles showing the flame temperature (solid line) and major species concentrations, $\mathrm{CO}_{2}$ (dashed line) and $\mathrm{CO}$ (dotted line), at standard conditions of $1 \mathrm{~atm}$ and $300 \mathrm{~K}$. Three equivalence ratios are shown with $\phi=0.6$ in (a), $\phi=1.0$ in (b) and $\phi=1.4$ in (c). Legend: mechanism predictions from Z69 in blue, LLNL in green [28], Aramco in red [17], K71 in magenta [32] and San Diego in black [30].

\section{Summary and Discussion}

In this work a reduced reaction mechanism for DME-air combustion is presented and validated for key flame properties over a wide range of conditions.

The reaction mechanism builds on the block structure modelling approach previously used to model a range of alkane and alkene fuels [35,43,49-51], with the approach consisting of a $\mathrm{H} / \mathrm{C}_{1} / \mathrm{O}$ submechanism of high complexity coupled to submechanisms for the fuel breakdown and intermediate hydrocarbon oxidation.

Overall, the agreements of the presented reaction mechanism to the reference mechanisms and the experimental data are good over the wide ranges of conditions investigated. The sensitivity analyses show that the reactions with the highest and lowest sensitivities are similar for all reaction mechanisms, indicating similar reactions, species and reaction pathways between their respective underlying chemistries. When considering that the 
three reduced reaction mechanisms mentioned in the introduction all use either reversible reactions only or a combination of reversible and irreversible reactions, the proposed Z69 contains 35\% fewer reactions than the smallest of the other three mechanisms. The low number of species and reaction makes Z69 highly suitable for three-dimensional CFD, where a small mechanism size is of utmost importance. Added to this, Z69 is also capable of predicting all the above-mentioned flame parameters, such as laminar burning velocity and ignition delay time, something that for example the Pan82 mechanism cannot.

This work presents good possibilities for including high-performing kinetics for DMEair combustion in finite rate combustion LES. Z69 builds on the same chemical kinetic platform as used in reaction mechanisms for other alkane and alkene fuels [35,36,43,49-51], solidifying the usability, versatility and flexibility of that platform and extending its use to also include oxygenated fuels. By including proper fuel breakdown and intermediate hydrocarbon submechanisms, it has been proven that the base mechanism can be used to predict key flame parameters, including ignition with NTC behaviour, for as diverse fuels as small oxygenated hydrocarbons (Z69) and large alkanes [51].

Author Contributions: Conceptualization, N.Z.; methodology, N.Z.; software, N.Z.; validation, N.Z., E.J.K.N. and C.F.; formal analysis, N.Z., E.J.K.N.; investigation, N.Z.; data curation, N.Z.; writingoriginal draft preparation, N.Z., E.J.K.N. and C.F.; writing-review and editing, N.Z., E.J.K.N. and C.F.; visualization, N.Z.; supervision, E.J.K.N., C.F.; project administration, C.F.; funding acquisition, C.F. All authors have read and agreed to the published version of the manuscript.

Funding: The presented work was supported by the Swedish Armed Forces, the Swedish Defence Material Agency, FMV, and the Swedish Defence Research Agency, FOI.

Conflicts of Interest: The authors declare no conflict of interest. The funders had no role in the design of the study; in the collection, analyses, or interpretation of data; in the writing of the manuscript, or in the decision to publish the results.

\section{Appendix A}

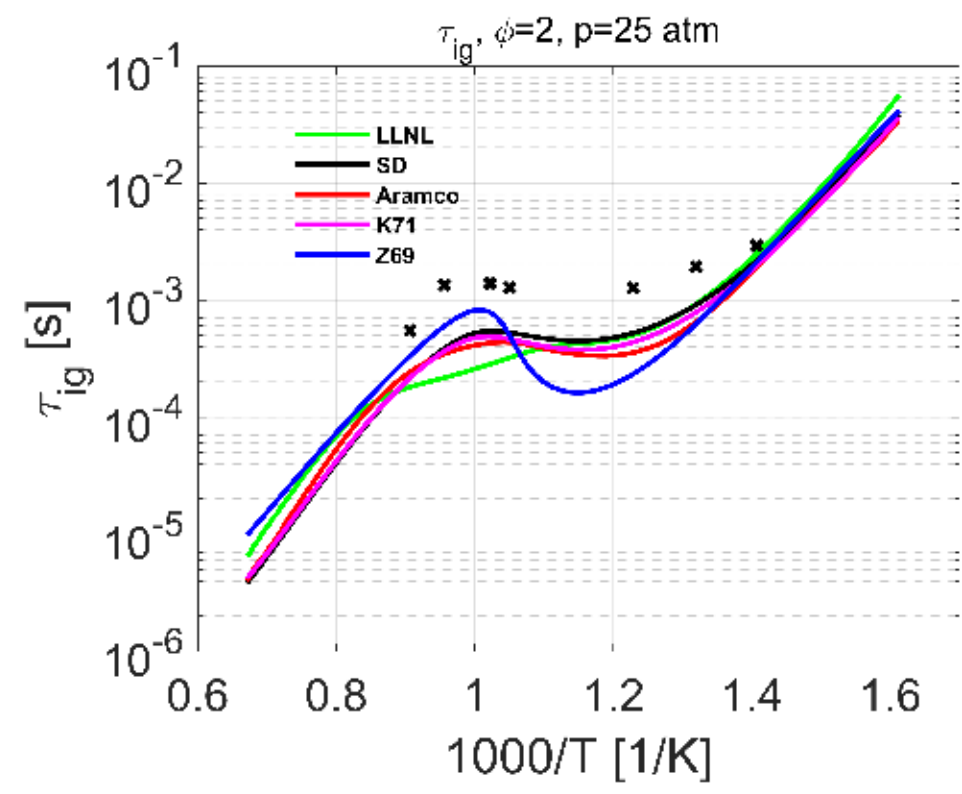

Figure A1. Ignition delay time for DME-air mixtures at 25 atm and $\phi=2.0$. Legend: experimental data for $\tau_{\text {ig }}$ from $(\times)$ Burke et al. [17]. Mechanism predictions from Z69 in blue, LLNL in green [28], Aramco in red [17], K71 in magenta [32] and San Diego in black [30]. 


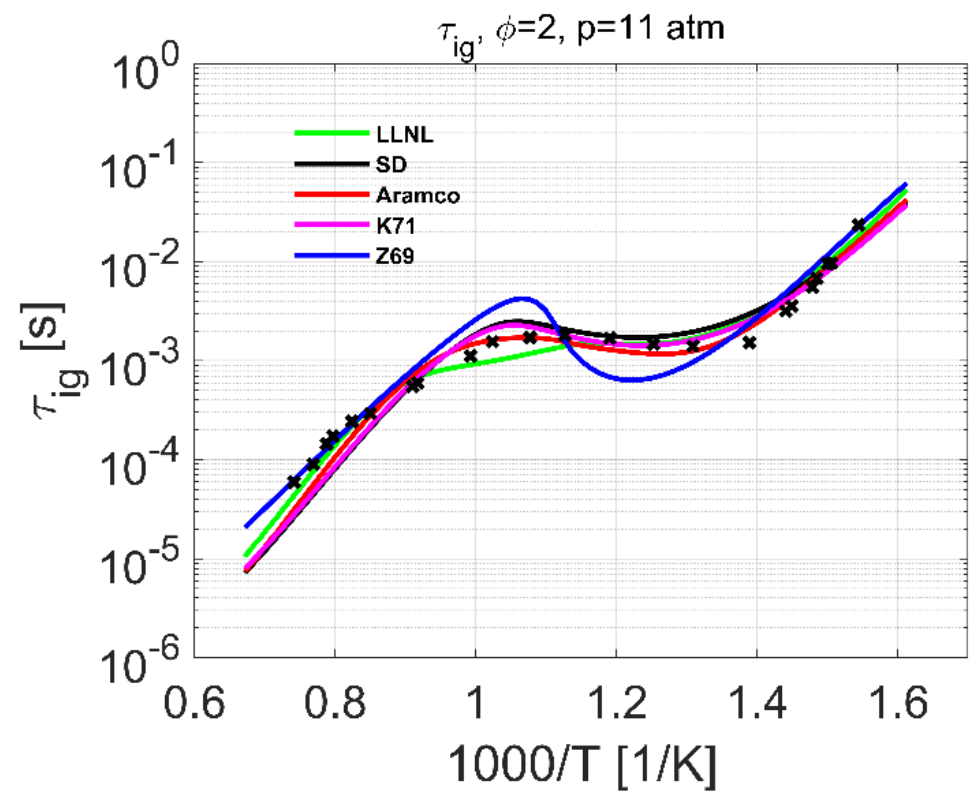

Figure A2. Ignition delay time for DME-air mixtures at $11 \mathrm{~atm}$ and $\phi=2.0$. Legend: experimental data for $\tau_{\text {ig }}$ from $(\times)$ Burke et al. [17]. Mechanism predictions from Z69 in blue, LLNL in green [28], Aramco in red [17], K71 in magenta [32] and San Diego in black [30].

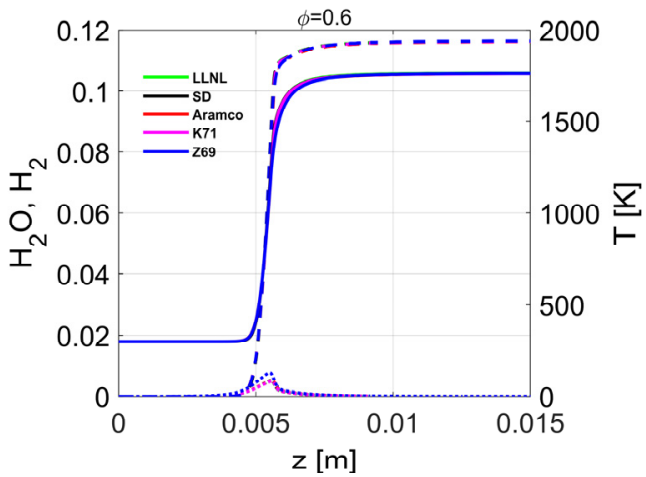

(a)

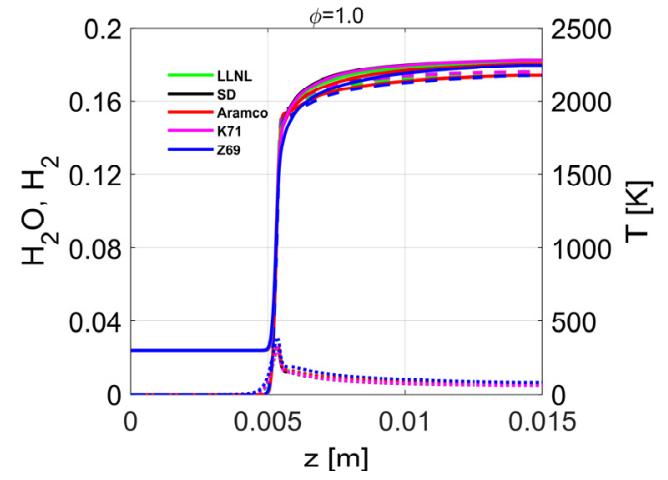

(b)

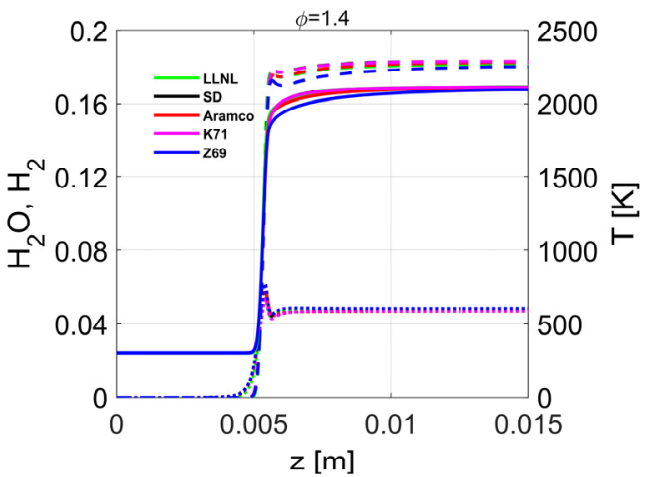

(c)

Figure A3. Flame profiles showing the flame temperature (solid line) and major species concentrations, $\mathrm{H}_{2} \mathrm{O}$ (dashed line) and $\mathrm{H}_{2}$ (dotted line), at standard conditions of $1 \mathrm{~atm}$ and $300 \mathrm{~K}$. Three equivalence ratios are shown with $\phi=0.6$ in (a), $\phi=1.0 \mathrm{in}(\mathbf{b})$ and $\phi=1.4$ in (c). Legend: mechanism predictions from Z69 in blue, LLNL in green [28], Aramco in red [17], K71 in magenta [32] and San Diego in black [30]. 


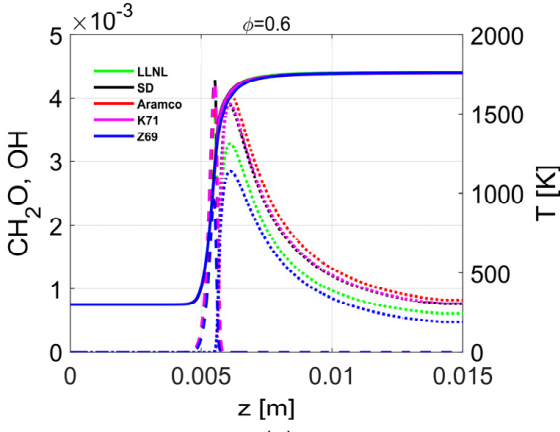

(a)

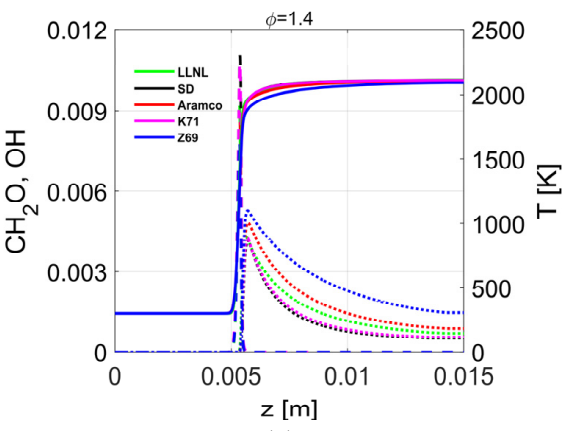

(c)

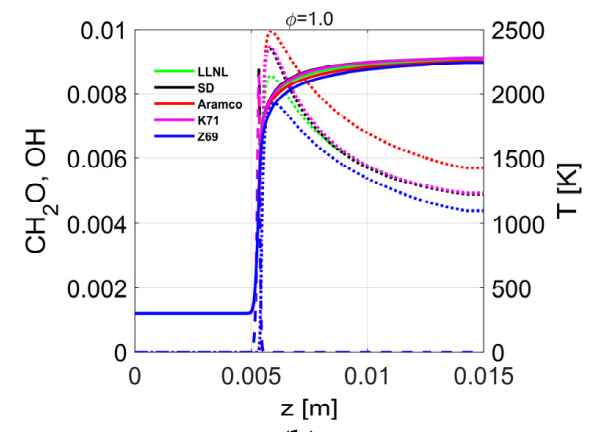

(b)

Figure A4. Flame profiles showing the flame temperature (solid line) and minor species concentrations, $\mathrm{CH}_{2} \mathrm{O}$ (dashed line) and $\mathrm{OH}$ (dotted line), at standard conditions of $1 \mathrm{~atm}$ and $300 \mathrm{~K}$. Three equivalence ratios are shown with $\phi=0.6$ in (a), $\phi=1.0$ in (b) and $\phi=1.4$ in (c). Legend: mechanism predictions from Z69 in blue, LLNL in green [28], Aramco in red [17], K71 in magenta [32] and San Diego in black [30].

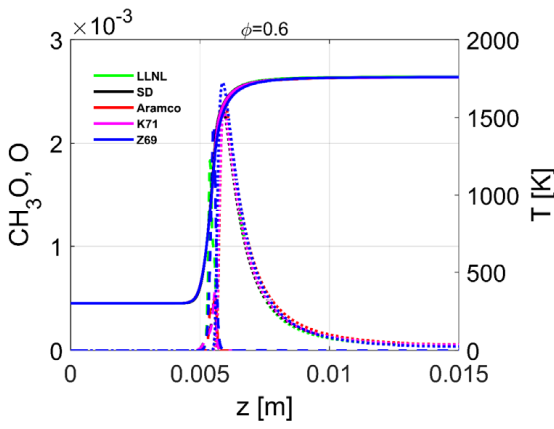

(a)

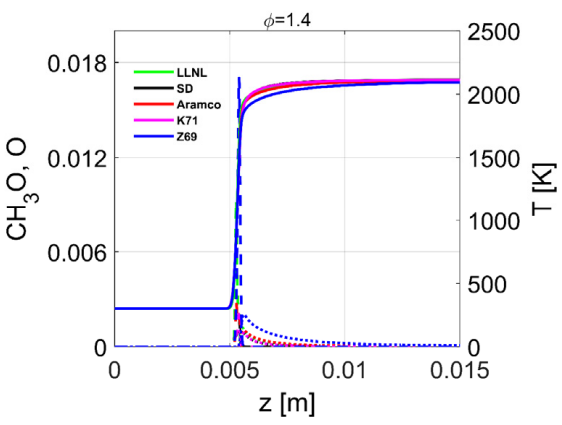

(c)

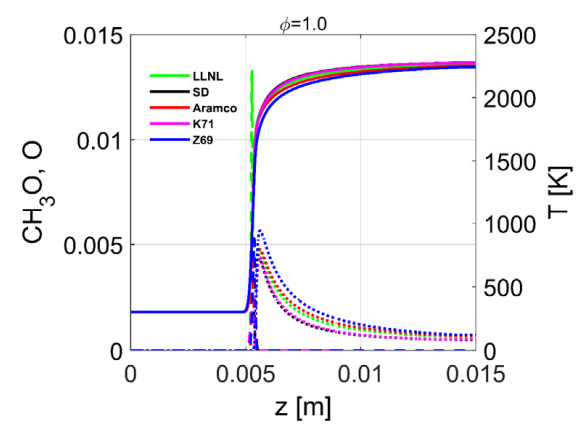

(b)

Figure A5. Flame profiles showing the flame temperature (solid line) and minor species concentrations, $\mathrm{CH}_{3} \mathrm{O}$ (dashed line) and $\mathrm{O}$ (dotted line), at standard conditions of $1 \mathrm{~atm}$ and $300 \mathrm{~K}$. The values for $\mathrm{CH}_{3} \mathrm{O}$ have been timed by 200 in order to be easier to read. Three equivalence ratios are shown with $\phi=0.6$ in (a), $\phi=1.0$ in (b) and $\phi=1.4$ in (c). Legend: mechanism predictions from Z69 in blue, LLNL in green [28], Aramco in red [17], K71 in magenta [32] and San Diego in black [30]. 


\section{References}

1. Semelsberger, T.A.; Borup, R.L.; Greene, H.L. Dimethyl Ether (DME) as an Alternative Fuel. J. Power Sources 2006, 156, 497-511. [CrossRef]

2. Ying, W.; Longbao, Z. Performance and Emissions of a Compression-Ignition Engine Fueled with Dimethyl Ether and Rapeseed Oil Blends. Energy Fuels 2007, 21, 1454-1458. [CrossRef]

3. Ying, W.; Genbao, L.; Wei, Z.; Longbao, Z. Study on the Application of DME/Diesel Blends in a Diesel Engine. Fuel Process. Technol. 2008, 89, 1272-1280. [CrossRef]

4. Ying, W.; Longbao, Z.; Hewu, W. Diesel Emission Improvements by the Use of Oxygenated DME/Diesel Blend Fuels. Atmos. Environ. 2006, 40, 2313-2320. [CrossRef]

5. Longbao, Z.; Hewu, W.; Deming, J.; Zuohua, H. Study of Performance and Combustion Characteristics of a DME-Fueled Light-Duty Direct-Injection Diesel Engine; SAE Technical Paper: Warrendale, PA, USA, 1999. [CrossRef]

6. Kapus, P.; Ofner, H. Development of Fuel Injection Equipment and Combustion System for DI Diesels Operated on Dimethyl Ether; SAE Technical Paper: Warrendale, PA, USA, 1995. [CrossRef]

7. Lee, M.C.; Seo, S.B.; Chung, J.H.; Joo, Y.J.; Ahn, D.H. Industrial Gas Turbine Combustion Performance Test of DME to Use as an Alternative Fuel for Power Generation. Fuel 2009, 88, 657-662. [CrossRef]

8. Kobayashi, N.; Inoue, H.; Koizumi, H. Robust Design of the Coaxial Jet Cluster Nozzle Burner for DME Fuel. In Proceedings of the ASME Turbo EXPO, Atlanta, GA, USA, 16-19 June 2003; p. 38410.

9. Gökalp, I.; Lebas, E. Alternative Fuels for Industrial Gas Turbines (AFTUR). Appl. Therm. Eng. 2004, 24, 1655-1663. [CrossRef]

10. Oh, C.; Jang, J.; Bae, C. The Effect of LPG Composition on Combustion and Performance in a DME-LPG Dual-Fuel HCCI Engine; SAE Technical Paper: Warrendale, PA, USA, 2010. [CrossRef]

11. Yeom, K.; Bae, C. Knock Characteristics in Liquefied Petroleum Gas (LPG)_dimethyl Ether (DME) and Gasoline-DME Homogeneous Charge Compression Ignition Engines. Energy Fuels 2009, 23, 1956-1964. [CrossRef]

12. Song, R.; Li, K.; Feng, Y.; Liu, S. Performance and Emission Characteristics of DME Engine with High Ratio of EGR. Energy Fuels 2009, 23, 5460-5466. [CrossRef]

13. Hu, J.; Wang, Y.; Cao, C.; Elliott, D.C.; Stevens, D.J.; White, J.F. Conversion of Biomass Syngas to DME Using a Microchannel Reactor. Ind. Eng. Chem. Res. 2005, 44, 1722-1727. [CrossRef]

14. Adachi, Y.; Komoto, M.; Watanabe, I.; Ohno, Y.; Fujimoto, K. Effective Utilization of Remote Coal through Dimethyl Ether Synthesis. Fuel 2000, 79, 229-234. [CrossRef]

15. Jiang, X.; Tian, Z.; Zhang, Y.; Huang, Z. Shock Tube Measurement and Simulation of DME/n-Butane/Air Mixtures: Effect of Blending in the NTC Region. Fuel 2017, 203, 316-329. [CrossRef]

16. Warnatz, J.; Maas, U.; Dibble, R.W. Combustion, 5th ed.; Springer: Berlin, Germany, 1999; Volume 26.

17. Burke, U.; Somers, K.P.; O'Toole, P.; Zinner, C.M.; Marquet, N.; Bourque, G.; Petersen, E.L.; Metcalfe, W.K.; Serinyel, Z.; Curran, H.J. An Ignition Delay and Kinetic Modeling Study of Methane, Dimethyl Ether, and Their Mixtures at High Pressures. Combust. Flame 2015, 162, 315-330. [CrossRef]

18. Pope, S.B. Small Scales, Many Species and the Manifold Challenges of Turbulent Combustion. Proc. Combust. Inst. 2013, 34, 1-31. [CrossRef]

19. Bourque, G.; Healy, D.; Curran, H.; Zinner, C.; Kalitan, D.; De Vries, J.; Aul, C.; Petersen, E. Ignition and Flame Speed Kinetics of Two Natural Gas Blends with High Levels of Heavier Hydrocarbons. In Proceedings of the ASME Turbo Expo 2008: Power for Land, Sea, and Air, Berlin, Germany, 9-13 June 2008.

20. Lieuwen, T.C.; Yang, V. Combustion Instabilities in Gas Turbine Engines: Operational Experience, Fundamental Mechanisms, and Modeling; AIAA: Danvers, MA, USA, 2005.

21. Daly, C.A.; Simmie, J.M.; Würmel, J.; Djeballi, N.; Paillard, C. Burning Velocities of Dimethyl Ether and Air. Combust. Flame 2001, 125, 1329-1340. [CrossRef]

22. Zhao, Z.; Kazakov, A.; Dryer, F.L. Measurements of Dimethyl Ether/Air Mixture Burning Velocities by Using Particle Image Velocimetry. Combust. Flame 2004, 139, 52-60. [CrossRef]

23. Qin, X.; Ju, Y. Measurements of Burning Velocities of Dimethyl Ether and Air Premixed Flames at Elevated Pressures. Proc. Combust. Inst. 2005, 30, 233-240. [CrossRef]

24. Jomaas, G. An Experimental Study on the Laminar Burning Velocities and Stability Boundaries of Outwardly Propagating Spherical Flames. Ph.D. Thesis, Princeton University, Princeton, NJ, USA, 2005.

25. Wang, Y.L.; Holley, A.T.; Ji, C.; Egolfopoulos, F.N.; Tsotsis, T.T.; Curran, H.J. Propagation and Extinction of Premixed DimethylEther/Air Flames. Proc. Combust. Inst. 2009, 32, 1035-1042. [CrossRef]

26. De Vries, J.; Lowry, W.B.; Serinyel, Z.; Curran, H.J.; Petersen, E.L. Laminar Flame Speed Measurements of Dimethyl Ether in Air at Pressures up to 10 Atm. Fuel 2011, 90, 331-338. [CrossRef]

27. Zhao, Z.; Chaos, M.; Kazakov, A.; Dryer, F.L. Thermal Decomposition Reaction and a Comprehensive Kinetic Model of Dimethyl Ether. Int. J. Chem. Kinet. 2008, 40, 1-18. [CrossRef]

28. Fischer, S.L.; Dryer, F.L.; Curran, H.J. The Reaction Kinetics of Dimethyl Ether. I: High-temperature Pyrolysis and Oxidation in Flow Reactors. Int. J. Chem. Kinet. 2000, 32, 713-740. [CrossRef]

29. Curran, H.J.; Fischer, S.L.; Dryer, F.L. The Reaction Kinetics of Dimethyl Ether. II: Low-temperature Oxidation in Flow Reactors. Int. J. Chem. Kinet. 2000, 32, 741-759. [CrossRef] 
30. "Chemical-Kinetic Mechanisms for Combustion Applications", San Diego Mechanism Web Page; Mechanical and Aerospace Engineering (Combustion Research), University of California at San Diego: La Jolla, CA, USA, 2011; Available online: http://combustion. ucsd.edu (accessed on 29 August 2018).

31. Bolshova, T.; Shvartsberg, V.; Dmitriev, A.; Knyazkov, D. Flame Structure and a Compact Reaction Mechanism for Combustion of Dimethyl Ether at Atmospheric Pressure. Fuel 2019, 255, 115752. [CrossRef]

32. Khare, R.S.; Parimalanathan, S.K.; Raghavan, V.; Narayanaswamy, K. A Comprehensively Validated Compact Mechanism for Dimethyl Ether Oxidation: An Experimental and Computational Study. Combust. Flame 2018, 196, 116-128. [CrossRef]

33. Pan, L.; Kokjohn, S.; Huang, Z. Development and Validation of a Reduced Chemical Kinetic Model for Dimethyl Ether Combustion. Fuel 2015, 160, 165-177. [CrossRef]

34. Gicquel, L.Y.; Staffelbach, G.; Poinsot, T. Large Eddy Simulations of Gaseous Flames in Gas Turbine Combustion Chambers. Prog. Energy Combust. Sci. 2012, 38, 782-817. [CrossRef]

35. Larsson, A.; Zettervall, N.; Hurtig, T.; Nilsson EJ, K.; Ehn, A.; Petersson, P.M.; Alden Larfeldt, J.; Fureby, C. Skeletal Methane-Air Reaction Mechanism for Large Eddy Simulation of Turbulent Microwave-Assisted Combustion. Energy Fuels 2017, 31, 1904-1926. [CrossRef]

36. Zettervall, N.; Fureby, C. A Computational Study of Ramjet, Scramjet and Dual-Mode Ramjet Combustion in Combustor with a Cavity Flameholder. In Proceedings of the 2018 AIAA Aerospace Sciences Meeting, Kissimmee, FL, USA, 8-12 January 2018; p. 1146.

37. Zettervall, N. Methodology for Developing Reduced Reaction Mechanisms, and Their Use in Combustion Simulations. Ph.D. Thesis, Lund University, Lund, Sweden, 2021.

38. Battin-Leclerc, F.; Simmie, J.M.; Blurock EBattin-Leclerc, F.; Simmie, J.M.; Blurock, E. Cleaner Combustion. Developing Detailed Chemical Kinetic Models. In Green Energy and Technology; Springer: Berlin, Germany, 2013.

39. Wang, H.; Xu, R.; Wang, K.; Bowman, C.T.; Davidson, D.F.; Hanson, R.K.; Bre-zinsky, K.; Egolfopoulos, F.N. A Physics-Based Approach to Modeling Real-Fuel Combustion Chemistry-I. Evidence from Experiments, and Thermodynamic, Chemical Kinetic and Statistical Considerations. Combust. Flame 2018, 193, 502-519. [CrossRef]

40. Bulat, G.; Fedina, E.; Fureby, C.; Meier, W.; Stopper, U. Reacting Flow in an Industrial Gas Turbine Combustor: LES and Experimental Analysis. Proc. Combust. Inst. 2015, 35, 3175-3183. [CrossRef]

41. Zettervall, N.; Fedina, E.; Nordin-Bates, K.; Heimdal Nilsson, E.; Fureby, C. Combustion LES of a Multi-Burner Annular Aeroengine Combustor Using a Skeletal Reaction Mechanism for Jet-A Air Mixtures. In Proceedings of the 51st AIAA/SAE/ASEE Joint Propulsion Conference, Orlando, FL, USA, 27-29 July 2015; p. 4020.

42. Zettervall, N.; Worth, N.A.; Mazur, M.; Dawson, J.R.; Fureby, C. Large Eddy Simulation of CH4-Air and C2H4-Air Combustion in a Model Annular Gas Turbine Combustor. Proc. Proc. Combust. Institute. 2019, 37, 5223-5231. [CrossRef]

43. Zettervall, N.; Nordin-Bates, K.; Nilsson EJ, K.; Fureby, C. Large Eddy Simulation of a Premixed Bluff Body Stabilized Flame Using Global and Skeletal Reaction Mechanisms. Combust. Flame 2017, 179, 1-22. [CrossRef]

44. Liu, B.; He, G.Q.; Qin, F.; An, J.; Wang, S.; Shi, L. Investigation of Influence of Detailed Chemical Kinetics Mechanisms for Hydrogen on Supersonic Combustion Using Large Eddy Simulation. Int. J. Hydrog. Energy 2019, 44, 5007-5019. [CrossRef]

45. Fureby, C. A Comparative Study of Subgrid Models, Reaction Mechanisms and Combustion Models in LES of Supersonic Combustion. In Proceedings of the AIAA Propulsion and Energy 2019 Forum, Indianapolis, IN, USA, 19-22 August 2019; Volume AIAA-4273, p. 4273.

46. Vincent-Randonnier, A.; Sabelnikov, V.; Ristori, A.; Zettervall, N.; Fureby, C. An Experimental and Computational Study of Hydrogen-Air Combustion in the LAPCAT II Supersonic Combustor. Proc. Combust. Inst. 2019, 37, 3703-3711. [CrossRef]

47. Vincent-Randonnier, A.; Sabelnikov, V.; Ristori, A.; Zettervall, N.; Fureby, C. A Combined Experimental and Computational Study of the LAPCAT II Supersonic Combustor. In Proceedings of the 22nd AIAA International Space Planes and Hypersonics Systems and Technologies Conference, Orlando, FL, USA, 17-19 September 2018; p. 5208.

48. Danel, K.; Zettervall, N.; Fureby, C. A Combined Experimental and Computational Study of Jet Engine Combustion-Baseline Engine Operation. In AIAA Propulsion and Energy; AIAA: Indianapolis, Indiana, 2019; p. 4328.

49. Zettervall, N.; Fureby, C.; Nilsson, E.J.K. Small Skeletal Kinetic Reaction Mechanism for Ethylene-Air Combustion. Energy Fuels 2017, 31, 14138-14149. [CrossRef]

50. Zettervall, N.; Fureby, C.; Nilsson, E.J.K. Small Skeletal Kinetic Mechanism for Kerosene Combustion. Energy Fuels 2016, 30, 9801-9813. [CrossRef]

51. Zettervall, N.; Fureby, C.; Nilsson, E.J.K. A Reduced Chemical Kinetic Reaction Mechanism for Kerosene-Air Combustion. Fuel 2020, 269, 117446. [CrossRef]

52. Smoke, M.D.; Giovangigli, V. Formulation of the premixed and nonpremixed test problems. In Reduced Kinetic Mechanisms and Asymptotic Approximations for Methane-Air Flames; Elsevier: Berlin/Heidelberg, Germany, 1991; pp. 1-28.

53. Ehn, A.; Zhu, J.J.; Petersson, P.; Li, Z.S.; Aldén, M.; Fureby, C.; Larfeldt, J. Plasma Assisted Combustion: Effects of O3 on Large Scale Turbulent Combustion Studied with Laser Diagnostics and Large Eddy Simulations. Proc. Combust. Inst. 2015, 35, $3487-3495$. [CrossRef]

54. Fureby, C.; Zettervall, N.; Kim, S.; Menon, S. Large Eddy Simulation of a Simplified Lean Premixed Gas Turbine Combustor. In Proceedings of the Ninth International Symposium on Turbulence and Shear Flow Phenomena, Melbourne, Australia, 3-30 June 2015. 
55. Glassman, I.; Yetter, R.A. Combustion, 4th ed.; Elsevier: Amsterdam, The Netherlands, 2010.

56. Goodwin, D.G.; Speth, R.L.; Moffat, H.K.; Weber, B.W. Cantera: An Object-Oriented Software Toolkit for Chemical Kinetics, Thermodynamics, and Transport Processes. Version 2.5.1. 2021. Available online: https://www.cantera.org (accessed on 4 August 2021).

57. Goos, E.; Burcat, A.; Ruscic, B. Extended Third Millenium Ideal Gas and Condensed Phase Thermochemical Database for Combustion with Updates from Active Thermochemical Tables; Elke Goos: Remchingen, Germany, 2015. 